\title{
A family of non-periodic tilings of the plane by right golden triangles
}

\author{
Nikolay Vereshchagin* \\ HSE University, Russian Federation.
}

September 6, 2021

\begin{abstract}
We study a family of substitution tilings with similar right triangles of two sizes which is obtained using the substitution rule introduced in [Danzer, L. and van Ophuysen, G. A species of planar triangular tilings with inflation factor $\sqrt{-\tau}$. Res. Bull. Panjab Univ. Sci. 2000, 50, 1-4, pp. 137-175 (2001)]. In that paper, it is proved this family of tilings can be obtained from a local rule using decorated tiles. That is, that this family is sofic.

In the present paper, we provide an alternative proof of this fact. We use more decorated tiles than Danzer and van Ophuysen (22 in place of 10). However, our decoration of supertiles is more intuitive and our local rule is simpler.
\end{abstract}

\section{Introduction}

Tilings and local rules. Assume that a finite family of polygons $\left\{P_{1}, \ldots, P_{k}\right\}$, called proto-tiles, is given. Isometric images of those polygons are called tiles. A tiling $T$ is a family of pair-wise non-overlapping tiles, which means that the interiors of the tiles are disjoint. A patch is a finite tiling. A patch $P$ is called a fragment of a tiling $T$, if $P$ is a subset of $T$. If the diameter of a patch (the maximal distance between points of its tiles) is at most $d$, then we call that patch a $d$-patch. In a similar way we define $d$-fragments.

Local matching rules govern how tiles may be attached to each other in a tiling. More specifically, a local rule is identified by a positive real $d$ and by a set of $d$-patches, whose members are called illegal patches. A tiling satisfies the local rule, if it does not include illegal patches as fragments. For instance, all polygons $P_{1}, \ldots, P_{k}$ may be unit squares with colored sides, and the local rule may require that tiles are attached side-to-side and the colors on the adjacent sides match (the so-called Wang tiles).

Aperiodic tile sets. The pair (a set of proto-tiles, a local rule) is called aperiodic if all tilings of the plane satisfying the local rule are non-periodic and such tilings exist.

Aperiodic sets of Wang tiles were used to prove the undecidability of Berger's Domino problem: find an algorithm that given a Wang tile set finds out whether that set tiles the entire plane [12].

${ }^{*}$ The article is supported by Russian Science Foundation (20-11-20203). 
Substitutions. The usual scheme to prove non-periodicity is based on the notion of a substitution. A substitution $\sigma$ is defined by a similarity ratio $\phi<1$ and a way to cut every polygon $P_{i}$ into a finite number of parts where each part is congruent to some polygon from the family $\left\{\phi P_{1}, \ldots, \phi P_{k}\right\}$. The substitution acts on tilings as follows. Given a tiling, for all $i$, we cut every tile $P_{i}$ from the tiling, as defined by the substitution. We obtain a tiling of the same set by tiles of smaller size. Then we apply to the resulting tiling some fixed pre-chosen homothety $H$ with the coefficient $1 / \phi$ to obtain a tiling with initial tiles $P_{1}, \ldots, P_{k}$. That homothety will be called the reference homothety in the sequel. We call the resulting tiling the decomposition of the initial tiling and denote it by $\sigma T$. The inverse operation is called composition. That is, a tiling $T$ is a composition of a tiling $T^{\prime}$ if $T^{\prime}=\sigma T$.

A supertile is a tiling, which can be obtained from an initial tile $P_{i}$ by applying decomposition several times. A supertile of the form $\sigma^{n} P_{i}$ is called a supertile of order $n$. Thus each supertile of order $n$ consists of several supertiles of order $n-1$.

Assume that the substitution and the local rule have the following properties:

P1. All supertiles satisfy the local rule.

P2. Every tiling $T$ of the plane satisfying the local rule has a unique composition satisfying the local rule ("the unique composition property"). This tiling is then denoted by $\sigma^{-1} T$.

In this case it is not hard to show that all tilings of the plane satisfying the local rule are non-periodic and that such tilings exist. This can be shown as follows.

Existence. By P1 each supertile satisfies local rule. Obviously the linear size of a supertile $\sigma^{n} P_{i}$ is $(1 / \phi)^{n}$ times larger than that of $P_{i}$. Hence there are tiling of arbitrary large parts of the plane satisfying local rule. By compactness arguments this implies that there are such tilings of the entire plane.

Non-periodicity. Assume that a tiling $T$ satisfying local rule has a non-zero period a, that is, $T+\mathbf{a}=T$. Then the vector $\phi \mathbf{a}$ is the period of $\sigma^{-1} T$. Indeed, let $H$ denote the reference homotethy. The decomposition of the tiling $\sigma^{-1} T+\phi \mathbf{a}$ is equal to the decomposition of $\sigma^{-1} T$ shifted by the vector $H \phi \mathbf{a}=\mathbf{a}$, that is, to $T+\mathbf{a}$. By our assumption, we have $T+\mathbf{a}=T$. Thus both $\sigma^{-1} T+\phi \mathbf{a}$ and $\sigma^{-1} T$ are compositions of $T$ and they both satisfy local rule. By P2 we then have $\sigma^{-1} T+\phi \mathbf{a}=\sigma^{-1} T$. Repeating the argument, we can conclude that the vector $\phi^{2} \mathbf{a}$ is a period of the tiling $\sigma^{-2} T$. In this way we can construct a tiling whose period is much smaller than the linear sizes of tiles, which is impossible.

This scheme was used to prove aperiodicity of many tile sets. Perhaps, the most famous example is Penrose-Robinson P4 tilings, where the set of proto-tiles consists of two isosceles triangles (see [11, 7]). Other famous examples are Ammann tilings (two L-shaped hexagonal tiles) and AmmannBeenker tilings (a rhombus and a square). For the definition of these tilings and for more examples we refer two the textbooks [1, 7] and to the Tilings Encyclopedia [8].

A similar approach was used to show non-periodicity of the famous Robinson tilings [12] with Wang tiles. Robinson's construction does not fit exactly the described framework, as in that construction supertiles of order $n$ are built from 4 supertiles of order $n-1$ and several proto-tiles. However, for the version of Robinson tilings from the paper [3], the proof of non-periodicity follows exactly the above pattern. In the tiling of [3], there are $2^{14}$ proto-tiles, which are unit squares, and every tile is cut in four smaller squares. 
Substitution tilings. A tiling $T$ is called a substitution tiling ${ }^{1}$ associated with substitution $\sigma$, if for each finite subset $P \subset T$ there is a supertile including $P$. The property $\mathrm{P} 1$ implies that every substitution tiling satisfies local rule. In some cases the reverse is also true. We will call this property P3:

P3. Every tiling of the plane satisfying the local rule is a substitution tiling associated with the substitution $\sigma$.

For instance, it happens that the family of Penrose-Robinson tilings coincides with the family of substitution tilings associated with the respective substitution. The same happens for Ammann A2 tilings, see [4].

Decorations and sofic tilings. Assume now that we are given only a substitution $\sigma$ acting on a set $\left\{P_{1}, \ldots, P_{k}\right\}$ of polygons and no local rule. Then it is natural to ask whether there is a local rule such that the properties $\mathrm{P} 1$ and $\mathrm{P} 2$ hold, or a local rule defining the family $L$ of substitution tilings associated with $\sigma$. In many cases there is no such local rule. In such cases we would like to find a decoration of the family $\left\{P_{1}, \ldots, P_{k}\right\}$ and a local rule for the decorated family of polygons. This means the following:

- each proto-tile is replaced by a finite number of proto-tiles of the same shape (we think that they have different colors);

- a local rule is defined for decorated tiles, let $\tilde{L}$ denote the family of all tilings of the plane satisfying that local rule;

- the family $L$ coincides with the family of tilings obtained from tilings $\tilde{T} \in \tilde{L}$ by removing colors.

If such a decoration and local rule exist, then we say that the initial family $L$ is sofic.

To prove that the given decoration and local rule for decorated tiles satisfy the last item, one usually defines a substitution $\tilde{\sigma}$ for decorated proto-tiles with the following properties: (1) each decorated proto-tile is cut exactly in the same way as prescribed by the initial substitution $\sigma$ (see an example in Fig. 1) and (2) the properties P1 and P3 hold for $\tilde{\sigma}$ and the local rule. To show that it works, assume that there are a decorated substitution $\tilde{\sigma}$ and a local rule for decorated tilings with properties $\mathrm{P} 1$ and $\mathrm{P} 3$.

In one direction this is trivial: assume that $T$ is obtained from a tiling $\tilde{T} \in \tilde{L}$ by removing colors and assume that $P$ is a finite subset of $T$. Then $P$ is obtained from a fragment $\tilde{P} \subset \tilde{T}$ by removing colors. By $\mathrm{P} 3$ the fragment $\tilde{P}$ occurs in a colored supertile $\tilde{\sigma}^{n} \tilde{P}_{i}$. Thus $P$ is included in the supertile $\sigma^{n} P_{i}$.

In the reverse direction: assume that $T \in L$. Then every finite $P \subset T$ occurs in a supertile $S$, and by property $\mathrm{P} 1$ it has a correct decoration, which means that the resulting decorated tiling $\tilde{P}$ is in $\tilde{L}$. Those decorations for different fragments $P$ may be inconsistent. Using compactness arguments, we can show that it is possible to choose consistent such decorations ${ }^{2}$

\footnotetext{
${ }^{1}$ We use here the terminology of [6]. Another name for substitution tilings, self-affine tilings, was used in [13].

${ }^{2}$ Here are more details. Consider the tree whose vertices are correct decorations of fragments of the form $\left\{F_{1}, F_{2}, \ldots, F_{i}\right\}$. Edges connect a decoration of a fragment $\left\{F_{1}, F_{2}, \ldots, F_{i}\right\}$ to a decoration of the fragment $\left\{F_{1}, F_{2}, \ldots, F_{i}, F_{i+1}\right\}$ whenever the decorations are consistent. This tree has arbitrary long branches. Any vertex of the tree has finitely many neighbors. By König lemma 9], the tree has an infinite branch, which provides a correct decoration of the entire tiling.
} 


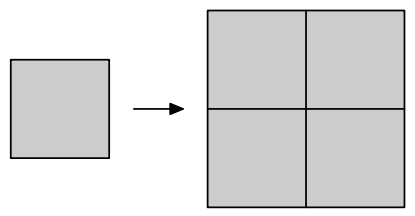

(a)
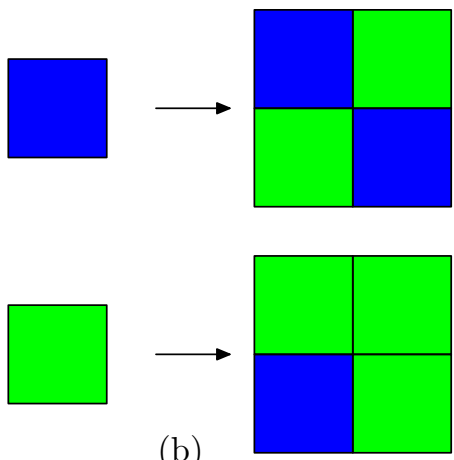

Figure 1: A substitution (a) and its decoration (b).

Note that to prove soficness, we do not need property P2 for the decorated family $\tilde{L}$. However, if we are not interested in proving that the initial family is sofic and our goal is just to construct an aperiodic tile set, we can use P2 (for $\tilde{L}$ ) instead of P3.

To construct a decoration and a local rule with properties P1 and P2, we can try to use a general Goodman-Strauss theorem [6] that claims that for every "good" substitution $\sigma$ there is a local rule for decorated tiles with properties $\mathrm{P} 1$ and $\mathrm{P} 23^{3}$ However, the resulting tile sets are generally gigantic and not really explicit. Besides, Goodman-Strauss theorem does not achieve the property P3. Both minor points of Goodman-Strauss theorem are inherited by its version by Fernique and Ollinger from [5]. In the case of tilings with Wang tiles, Mozes [10] proved in a seminal paper that the set of tilings generated by rectangular substitutions satisfying a particular property is sofic.

This paper. In this paper, we consider tilings with right "golden" triangles and the substitution introduced by Danzer and van Ophuysen [2]. Danzer and van Ophuysen showed that there is no local rule with properties P1 and P2 for that substitution and defined a decoration of the substitution and a local rule for decorated tilings with properties P1, P2 and P3. However, their decoration of the substitution is not intuitive and the local rule is complicated. The local rule uses the notion of a crown. Let $T$ be a tiling and $A$ a vertex of a tile from $T$. The crown centered at vertex $A$ within $T$ is a fragment of $T$ consisting of all tiles from $T$ that include the point $A$ (not necessarily as a vertex). The local rule of Danzer and van Ophuysen stipulates that every crown in the tiling occurs in a supertile. There are 65 such crowns (up to isometry) and the paper does not even provide their list.

The goal of the present paper is to provide a more intuitive decoration of the substitution and a simpler local rule for decorated tilings, also having properties P1, P2 and P3.

\section{Tilings with golden right triangles}

In this paper, we consider a specific substitution and the associated family of substitution tilings with right "golden" triangles introduced by Danzer and van Ophuysen [2] and later in [14].

\footnotetext{
${ }^{3}$ Goodman-Strauss formulates property P2, as "the local rule enforce the hierarchical structure associated with $\sigma$ ", which means that every tiling satisfying the local rule can be uniquely partitioned into supertiles of order $n$ for each $n$.
} 
Golden right triangles and tilings. The altitude of any right triangle cuts it into two similar triangles. Those triangles are denoted by $S, L$ in Fig. 2(a). If the angles of the original right triangle

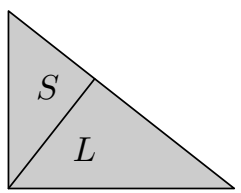

(a)

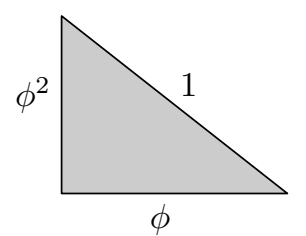

(b)

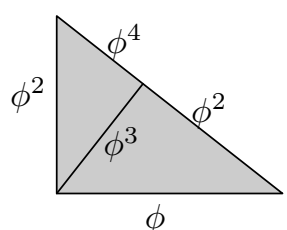

(c)

Figure 2: Golden right triangles.

are chosen appropriately, then the ratio of the size of the initial triangle to the size of $L$ equals the ratio of the size of $L$ to the size of $S$. More specifically, the ratio of the legs of the initial triangle should be equal to the square root of the golden ratio $\phi=\sqrt{\frac{\sqrt{5}-1}{2}}$. Such a triangle is shown in Fig. 2(b). The lengths of the sides of triangles $S, L$ are shown in Fig. 2(c). We will call triangles of this shape golden right triangles 4

We will use triangles $L$ and $S$ as proto-tiles. More specifically, isometric images of $L$ are called large tiles, and isometric images of $S$ are called small tiles. In Fig. 3, we can see an example of a tiling. We denote by $[T]$ the union of all tiles from $T$ and say that $T$ tiles $[T]$, or that $T$ is a tiling
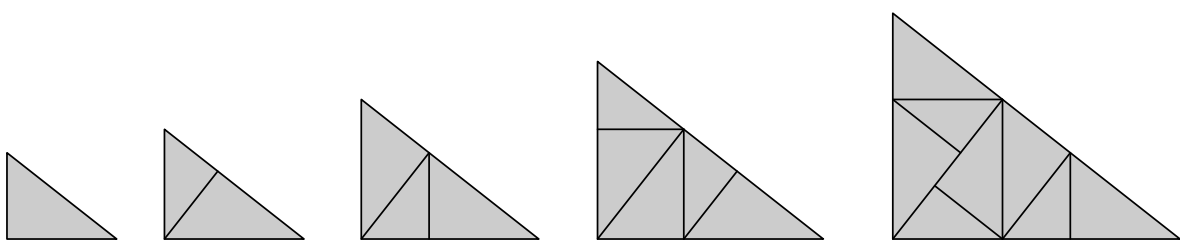

Figure 3: A tiling, which is a union of supertiles of orders $0,1,2,3,4$.

of $[T]$.

The substitution, decomposition and composition of tilings. We consider the following substitution:
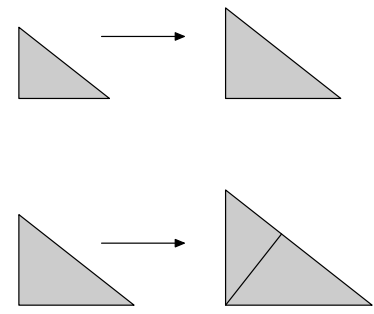

In the course of decomposition for this substitution, each large tile produces a large and a small tile in the decomposed tiling and each small tile becomes a large tile.

\footnotetext{
${ }^{4}$ The name "golden" in a similar context was used to call isosceles triangles whose all angles are integer multiples of $36^{\circ}$ (Robinson triangles). To avoid confusion, we add the attribute "right".
} 
As usual, we call a tiling $T$ a composition of a tiling $T^{\prime}$ if $T^{\prime}$ is the decomposition of $T$. There are tilings that have no composition, for instance, the tiling consisting of a single small tile. On the other hand, every tiling has at most one composition (the unique composition property). As usual, the composition of a tiling $T$ (if exists) is denoted by $\sigma^{-1} T$.

It may happen that the composition of a tiling again has a composition. In this case the initial tiling is called doubly composable. If a tiling can be composed any number of times, we call it infinitely composable. In terms of [6], infinitely composable tilings are those that have "hierarchical structure".

Supertiles. Recall that a supertile is a tiling, which can be obtained from a small or a large tile by applying decomposition several times. Since every large tile is a decomposition of a small tile, every supertile can be obtained from a small tile by applying decomposition some $n$ times. The number $n-1$ is called then the order of the supertile. (In particular, the small tile is a supertile of order -1.) Supertiles of order $i$ are denoted by $S_{i}$. Fig. 3 shows supertiles of orders $0,1,2,3,4$.

Substitution tilings. Recall that a tiling $T$ is called a substitution tiling if for each finite $P \subset T$ there is a supertile $S$ including $P$. For instance, all supertiles are substitution tilings. There exist substitution tilings of the entire plane. This can be deduced by compactness arguments from the existence of substitution tilings of arbitrarily large parts of the plane. However, it is easier to prove this using the following argument. There are supertiles of orders 0 and $8, S_{0}, S_{8}$, such that $S_{0} \subset S_{8}$ and $\left[S_{0}\right]$ is included in the interior of $\left[S_{8}\right]$. Indeed, in Fig. 4 we can see a supertile $T$ of order 8 . The interior of the triangle $[T]$ includes a large tile $A$ (shown in green color). Applying

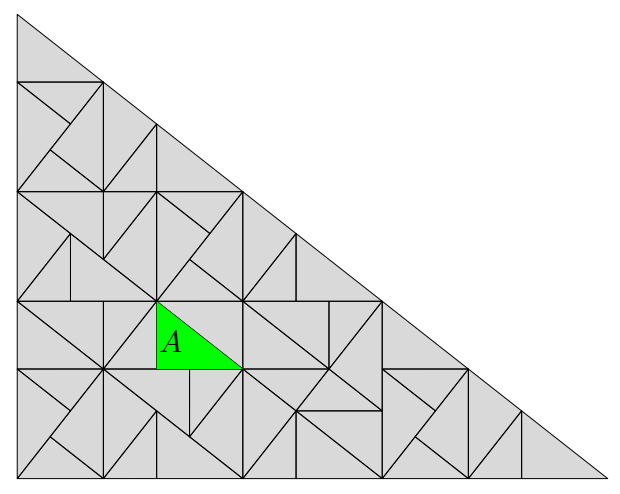

Figure 4: The green triangle $A$ is strictly inside a supertile of order 8 .

8 decompositions to the supertiles $\{A\}$ and $T$ we get supertiles $S_{8}=\sigma^{8}\{A\}$ and $S_{16}=\sigma^{8} T$, of orders 8 and 16, respectively. Since $A \in T$, we have $S_{8}=\sigma^{8}\{A\} \subset \sigma^{8} T=S_{16}$. In this way we can construct a tower of supertiles

$$
S_{0} \subset S_{8} \subset S_{16} \subset S_{24} \subset \ldots
$$

where each set $\left[S_{8 n}\right]$ extends the previous set $\left[S_{8(n-1)}\right]$ in all directions. Therefore the tiling $S_{0} \cup$ $S_{8} \cup S_{16} \cup \ldots$ tiles the entire plane and is a substitution tiling by construction.

It is not hard to see that every substitution tiling of the plane has a composition, which is again a substitution tiling. Thus every substitution tiling of the plane is infinitely composable. 
In particular, every substitution tiling of the plane contains supertiles of all orders. (To find a supertile of order $n$ in a substitution tiling $T$ of the plane, we can compose it $n$ times and then pick any large tile in the resulting tiling. The $n$-fold decomposition of that tile is a supertile of order $n$ and is included in the original tiling T.) As mentioned in the Introduction, the unique composition property implies that any infinitely composable (and hence any substitution) tiling of the plane is non-periodic.

In [2] and later in [14], it was shown that there is no local rule such that the family of tilings of the plane satisfying that local rule coincides with the family of substitution tilings. More specifically, it was proved that for any positive $d$ there is a periodic (and hence not substitution) tiling $T_{d}$ of the plane, whose all $d$-fragments occur in supertiles. For any local rule consisting of $d$-patches, either all $d$-patches from $T_{d}$ are declared legal, or a $d$-patch from $T_{d}$ is declared illegal. In the first case, the tiling $T_{d}$ satisfies the local rule. In the second case, some supertile has an illegal patch, and since all substitution tilings include that supertile, all substitution tilings do not satisfy the local rule.

We will outline a proof of this. We start with the periodic tiling $T$ shown in Fig. 5 . It has the

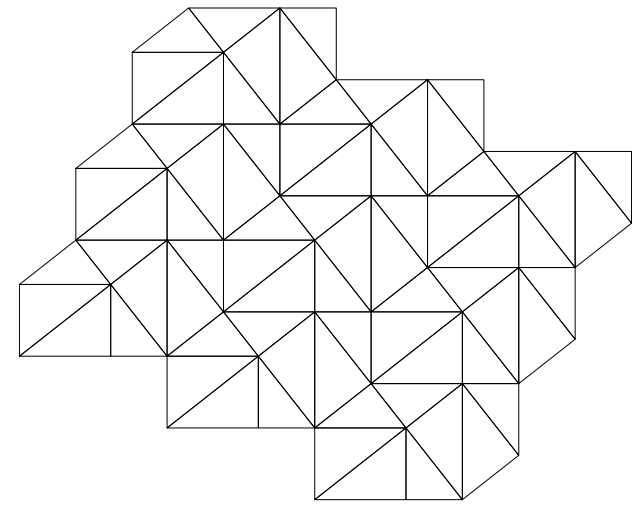

Figure 5: Periodic tiling $T$.

following remarkable feature: all its crowns occur in supertiles. In fact, up to an isometry, this tiling has a single crown, which occurs in the supertile of order 6 (see Fig. 4). If $k$ is large enough compared to $d$, then for any $d$-fragment $P$ of the tiling $\sigma^{k} T$ there is a single crown $C$ in $T$ such that $\sigma^{k} C$ includes the entire patch $P$ (this follows from Lemma 1 below). As we have seen, the crown $C$ is in the supertile $S_{6}$ and hence $\sigma^{k} C$ is in $S_{6+k}$. Thus all $d$-patches in $\sigma^{k} T$ occur in $S_{6+k}$ and we can let $T_{d}=\sigma^{k} T$.

\section{Tilings with decorated tiles}

In this section we show that the family of substitution tilings associated with our substitution is sofic.

\subsection{The local rule of Danzer and van Ophuysen}

We color both tiles in five colors $0,1,2,3,4$. The substitution $\tilde{\sigma}$ acts on decorated tiles as follows: 


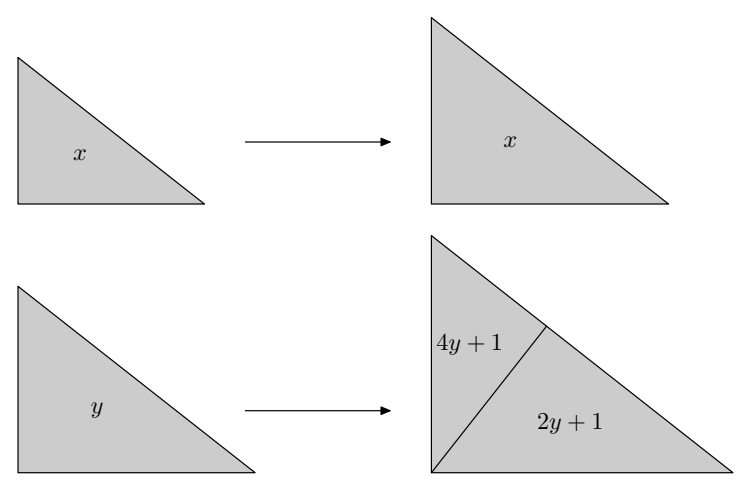

Addition and multiplication refer to the respective operations modulo 5.

The local rule is based on the notion of a legal crown. By definition, a crown is legal if it occurs in a supertile. The local rule stipulates that all crowns in the tiling must be legal. To make this local rule explicit, we need to list all legal crowns. There are 65 of them (up to an isometry), thus the list is quite long. However, we can reduce the list using the following observation.

Let us define on tilings the following operation called shift. To shift a tiling by $y=0,1,2,3,4$, we increment the markings of all large tiles by $y$ and the markings of all small tiles by $2 y$ (modulo 5 ). It is not hard to see that the shift of any legal crown is legal. Indeed, let $C$ be a crown within a supertile $S_{i}$ of order $i$, that is obtained from a large tile with color $k$. By induction on $i$ it is easy to prove the following: for each tile $A$ from $S_{i}$, its color is obtained from $k$ by applying a linear function of the form $2^{i+1} x+c_{A}$ for small tiles and $2^{i} x+c_{A}$ for large tiles. Here $c_{A}$ denotes a number depending on the location of $A$ within $S_{i}$. Thus, if we increase $k$ by $3^{i} y$, the colors of all large tiles are increased by $y$ and the colors of all small tiles by $2 y$, as $2 \cdot 3 \equiv 1(\bmod 5)$.

Let us call two legal crowns equivalent if they can be obtained from each other by a shift. Obviously each equivalence class has 5 legal crowns and hence there are 13 equivalence classes denoted $C_{1}, C_{2}, C_{3}, C_{4}, C_{5}, C_{6}, C_{7}$ and $C_{1}^{\prime}, C_{2}^{\prime}, C_{3}^{\prime}, C_{4}^{\prime}, C_{5}^{\prime}, C_{6}^{\prime}$. In Fig. 6 we present one legal crown from each equivalence class.

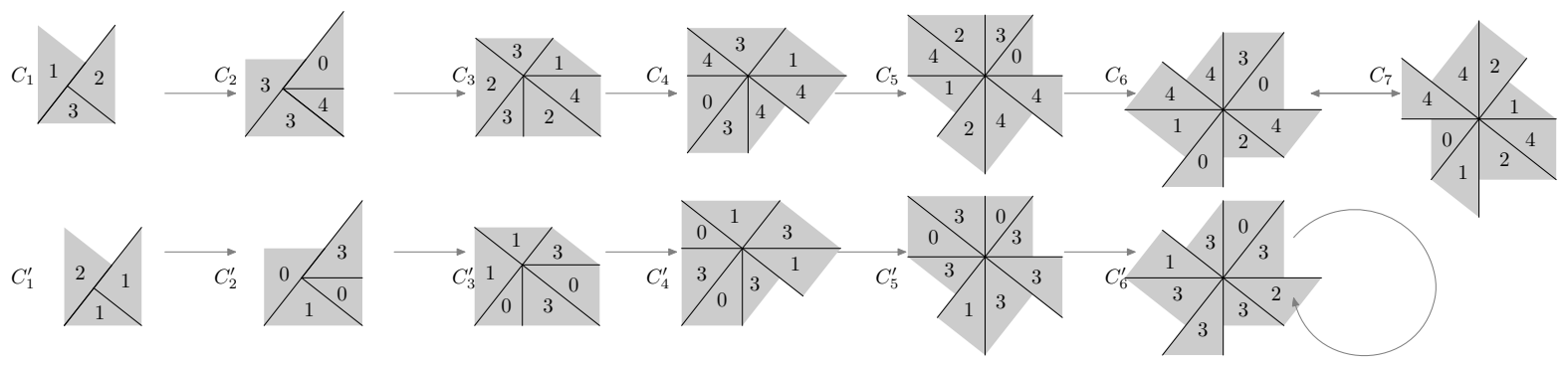

Figure 6: Legal crowns for Danzer and van Ophuysen substitution. Every crown represents 5 crowns obtained by shifts from it. Arrows indicate the action of substitution.

This local rule guarantees all the above properties P1, P2 and P3.

\subsection{Our local rule}

We define first how we color proto-tiles. We first choose for every side of the tile its orientation (depicted by an arrow). Besides, every side is labeled by an integer number from 0 to 3 . There 

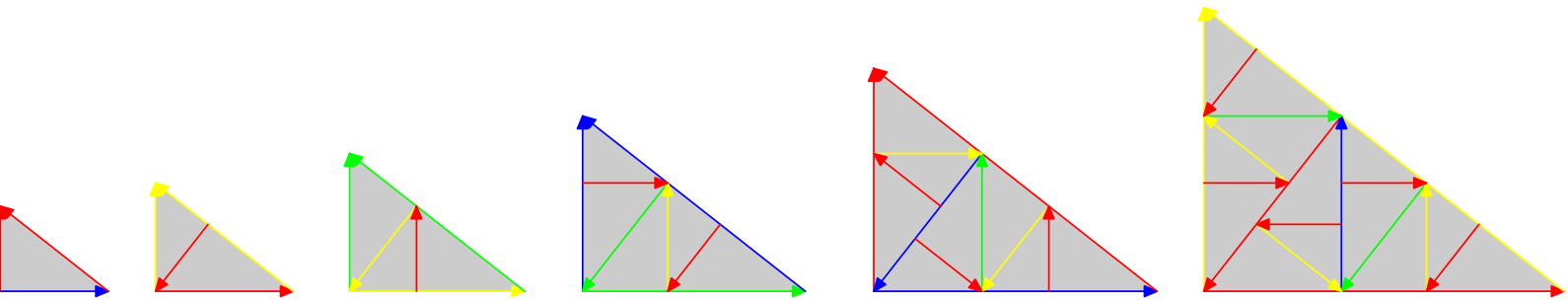

Figure 7: Colored supertiles $S_{0}, S_{1}, S_{2}, S_{3}, S_{4}, S_{5}$.

is the following restriction for those labels: the hypotenuse and the small leg of the large triangle, and the large leg of the small triangle have even labels, and the remaining sides have odd labels. Tiles bearing orientation and digital labels on sides are called colored tiles. Each of 2 proto-tiles produces $2^{3} \cdot 2^{3}=64$ colored proto-tiles. Actually, only 22 of these 128 tiles can occur in supertiles and hence we can reduce the number of proto-tiles to 22 . We will not prove this, since anyway we obtain more proto-tiles than Danzer and van Ophuysen.

Decomposition and composition of colored tilings. The substitution is extended to colored tiles as follows:

- for small tiles: we increment all digital labels by 1 modulo 4 and keep orientation of all sides

- for large tiles: we first increment all digital labels by 1 modulo 4 keeping orientation of all sides, and then we label the newly appeared altitude by 0 and orient it from the foot to the vertex. The axis of the altitude is divided into two segments, those segments keep their labels and orientations.

It is not hard to verify that the requirement of evenness/oddness of labels is preserved and thus we obtain again a tiling by legally colored tiles.

In Fig. 7 we have shown a large colored tile, its decomposition, the decomposition of its decomposition and so on. The digital labels are represented by colors: red is 0, yellow is 1, green is 2 , blue is 3 , orientations are shown by arrows. Long line segments of the same color represent identically oriented sides with the same digital label. This orientation is shown by an arrow at an end of the segment. The inverse operation is called the composition of colored tilings.

A colored supertile of order $n$ is defined as the $n$-fold decomposition of a large colored tile. Colored supertiles of orders $0,1,2,3,4,5$ are shown in Fig. 7. A tiling with decorated tiles is called a substitution tiling if every its fragment occurs in a (colored) supertile.

The intuition behind our decoration of supertiles. Each supertile $S_{i}, i>0$, sends a signal whose color equals $(i-1) \bmod 4$ along its altitude from its foot to the top. If supertiles $S_{n-1}$ and $S_{n}$ form a supertile $S_{n+1}$, then the vertex of the right angle of $\left[S_{n+1}\right]$ receives two signals, $(n-2) \bmod 4$ and $(n-1) \bmod 4$, and in turn sends the signal $n \bmod 4$. The local rule will ensure that all these three signals are "coherent", that is, are equal to $(i-2) \bmod 4,(i-1) \bmod 4, i \bmod 4$ for some $i$. If we were allowed infinitely many colors, the signal sent by $S_{i}$ would be just $i-1$. In that case the proof would be much easier. Each supertile $S_{n}$ has hierarchical structure, that is, for each $i<n$ it can be partitioned into supertiles $S_{i}$ and $S_{i-1}$. Hence $S_{n}$ hosts many signals. The crucial point is 

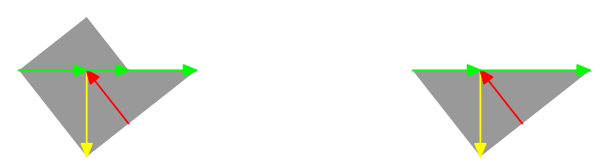

Figure 8: A complete star and an incomplete star. The colors and orientations of outer sides are not shown, as this information is not included in the star.
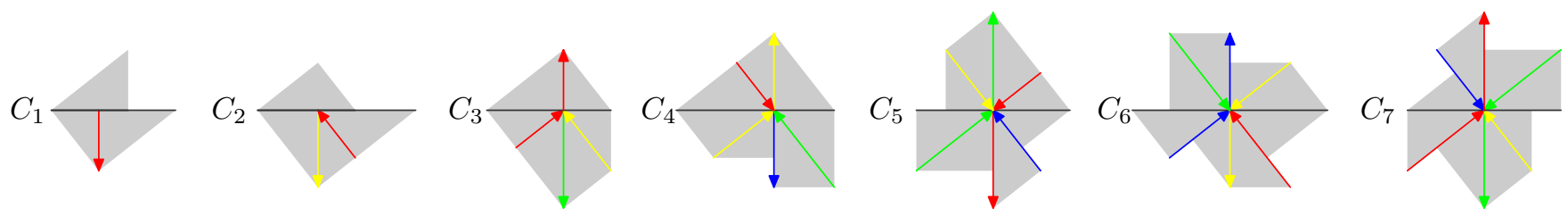

Figure 9: The list of legal stars.

that all those signals are sent along non-overlapping paths. We will explain later why the number of colors is 4 (see Remark 4 ).

Our local matching rule L. To define our local rule, we need a new notion, similar to that of a crown. We call this notion a star. Let $A$ be a vertex of a tile from a tiling $T$. Consider all non-decorated tiles from $T$ that include the point $A$ together with digital marks and orientations of all the sides that include the point $A$. That information forms the star within $T$ centered at $A$. It is important that we forget orientations and digital labels of the outer sides of tiles from a star. A star may be incomplete, which means that no neighborhood of $A$ is included in the union of tiles from the star. Incomplete stars appear on the borders of tilings of parts of the plane. Two examples of stars are shown in Fig. 8 .

Definition 1. A complete star is called legal if it is one of the stars shown in Fig. 9. The black line segment on that figure is called the axis of a legal star and may have any orientation and any color. All digital labels and orientations of all sides lying on the axis must coincide. An example of a legal star is shown in Fig. 8 on the left.

Definition 2. A tiling of the plane with decorated tiles satisfies our local rule L if (1) any two sides that share a common interval have matching orientations and digital labels and (2) every its star is legal. For a tiling of a part of the plane the second item reads: every its complete star is legal. Tilings satisfying the local rule $\mathrm{L}$ are called L-tilings. A coloring of tiles in a tiling with non-decorated tiles is called correct if the resulting tiling is an L-tiling.

\section{Several remarks on legal stars.}

Remark 1. We will prove that a star is legal if and only if it is a complete star within a supertile.

Remark 2. Observing the tiles in the legal stars we can easily conclude that the parity of the digital label of the axis of a legal star must be equal to that of the index of the star. Hence every star from Fig. 9 represents 4 legal stars: there are two ways to choose orientation of the axis and two ways to label it. Thus there are $7 \cdot 2 \cdot 2=28$ different legal stars, up to an isometry. 
Remark 3. There are one or two outgoing arrows from the center of any legal star and those arrows are orthogonal to the axis of the star. All the remaining arrows are directed towards the center of the star and form with the axis the acute angles $\arcsin ((\sqrt{5}-1) / 2)$ and $\arccos ((\sqrt{5}-1) / 2)$, called the smaller and the larger ones, respectively. Let $n$ denote the index of a legal star. Then the digital labels of the arrows that go into or out of the center of a legal star are the following. On one side of the star the arrow that goes into the center of the star and forms with the axis the smaller acute angle (if any) is labeled by $n+1$, the arrow that goes into the center of the star and forms with the axis the larger acute angle (if any) is labeled by $n+2$, and the outgoing arrow (if any) is labeled by $n+3$ (addition modulo 4 ). On the other side of the axis the digital labels are $n-1, n$ and $n+1$, respectively.

\section{Results}

The following three theorems claim that our decoration and local rule have the properties P1, P2 and P3.

Theorem 1. (1) Decomposition of any L-tiling of the plane is again an L-tiling. (2) Every supertile is an L-tiling. (3) Conversely, all legal stars occur in supertiles.

Corollary 2. There exists an L-tiling of the plane.

Theorem 3. Any L-tiling of the plane has a composition, which is again an L-tiling.

Theorem 4. A tiling of the plane with colored tiles is a substitution tiling if and only if it is an L-tiling.

It follows from Theorem 3 that all L-tilings of the plane are non-periodic. Indeed, they are infinitely composable, and hence non-periodic, as explained above. We first prove Theorem 1 and Corollary 2 , then we derive Theorem 4 from Theorem 3 and then we prove the latter.

Proof of Theorem 1. Let us extend decomposition to stars: to decompose a star, we decompose the respective tiling and then delete all the resulting tiles that do not include the center of the star. It is not hard to verify that the family of legal stars is closed under decomposition: see Fig. 10 where the action of decomposition is shown by grey arrows.

(1) Let $T$ be an L-tiling of the plane and $A$ a vertex of a tile from $\sigma T$. We have to show that the star centered at $A$ in $\sigma T$ is legal. We consider two cases.

Case 1: $A$ is also a vertex of a tile from $T$. Then the star centered at $A$ in $T$ is legal, as $T$ is an L-tiling. The star centered at $A$ in $\sigma T$ is obtained by decomposition from that star and hence is legal as well.

Case 2: $A$ is not a vertex of a tile from $T$. Then $A$ is a foot of the altitude of a large tile $F$ from $T$ and hence lies on the hypotenuse of $F$. Let $B, C$ denote endpoints of that hypotenuse (see Fig. 11). Consider the star within $T$ centered at $B$. Observing Fig. 9, we can see that such situation (the center of the star is an endpoint of the hypotenuse of a large tile and the foot of the altitude of that tile is not a vertex) occurs only in stars $C_{3}-C_{7}$ and in all cases the star includes the triangle $\tilde{F}$ obtained by the central symmetry centered at the middle point of the hypotenuse: $F$ together with $\tilde{F}$ from a rectangle. Decomposition of that rectangle produces two stars $C_{1}$.

(2) We use induction on the order of the supertile. Base of induction is trivial, since supertiles of order less than 4 have no complete stars. To make the induction step, we would like to extend 


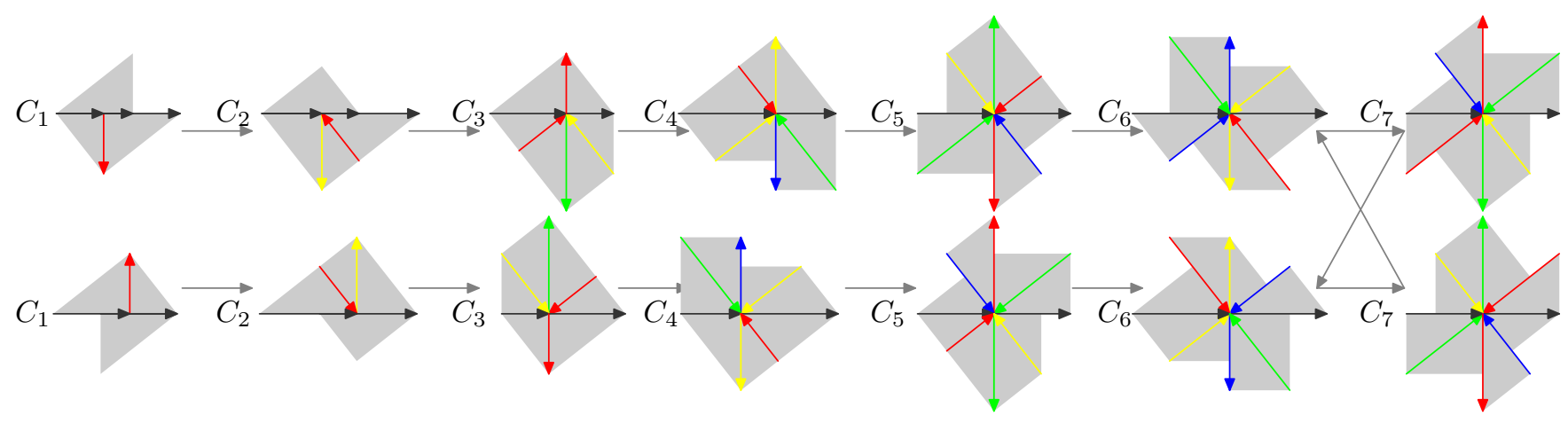

Figure 10: The action of decomposition on legal stars is shown by grey arrows. The stars $C_{6}, C_{7}$ in the course of 4 decompositions are mapped to themselves, since the decomposition works as rotation by the right angle (ignoring colors) on the stars $C_{6}, C_{7}$, and we increment digital labels modulo 4. Note that the decomposition maps both stars $C_{5}$ and $C_{7}$ to $C_{6}$. This is not surprising, as the stars $C_{5}$ and $C_{7}$ differ only in one tile and after decomposition this difference disappears.

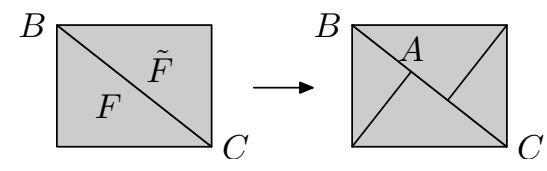

Figure 11: $A$ is not a vertex of a tile from $T$

item (1) to tilings of parts of the plane. However this cannot be done, as there is a tiling of a part of the plane with no complete stars such that its decomposition has a complete illegal star (see Fig. 12).

Why cannot we repeat the above arguments to show that decomposition of any L-tiling is an L-tiling? The only reason why the above arguments fail, is that in Case 2 we cannot claim that the star within $T$ centered at $B$ is legal and hence the tiling $T$ includes the large tile $\tilde{F}$. Indeed, it might happen, that although $A$ is an inner point of $[T]$, neither $B$, nor $C$ are inner points of $[T]$. In that case we have no information about the stars of $T$ centered at $B$ and $C$.

To handle this case, we will show that if $T$ is a supertile, $T=S_{i}$, then

(*) ignoring orientations and digital marks, all stars in $T=S_{i}$, including incomplete ones, can be extended to legal stars by adding some tiles that do not overlap tiles in $T$.

This statement can be proved by induction on $i$. Base of induction: the tile $S_{0}$ has three stars and they all can be extended to legal stars, provided we ignore digital marks and orientation 5

The induction step follows from the fact that the family of tilings $T$ satisfying $(*)$ is closed under decomposition. Indeed, assume that a tiling $T$ satisfies $\left(^{*}\right)$. If $A$ is a vertex in $T$, then the star centered at $A$ in $\sigma T$ can be extended to decomposition of the completion of the star of $A$ in $T$. Otherwise $A$ is a foot of the altitude of a large tile $F$ from $T$ and hence lies on the hypotenuse of $F$. Again we consider $B, C$, the endpoints of that hypotenuse, see Fig. 11. By our assumption, the

\footnotetext{
${ }^{5}$ This ignoring is important, as there are colored large tiles which possess stars that cannot be extended to legal stars.
} 


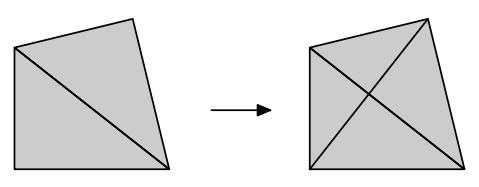

Figure 12: An L-tiling of a part of the plane whose decomposition is not an L-tiling

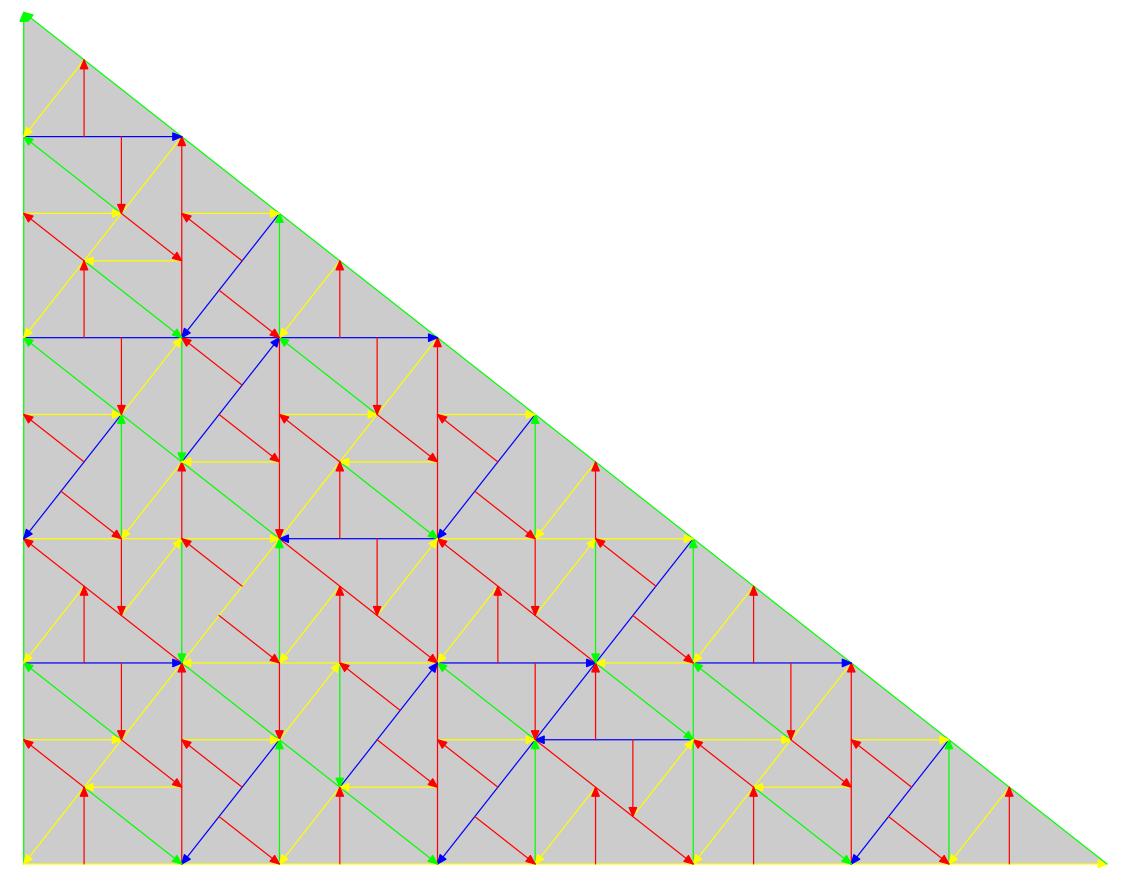

Figure 13: A colored supertile $S_{10}$ has all four starts of the type $C_{1}$.

star centered at $B$ in $T$ can be completed to a legal star (ignoring digital marks and orientation) by adding tiles that do not overlap $T$. As verified above, that star contains the triangle $\tilde{F}$, as shown in Fig. 11. Hence the star centered at $A$ in $\sigma T$ can be completed to the star $C_{1}$ by adding the large tile obtained by decomposition of $\tilde{F}$ (obviously, that tile does not overlap $\sigma T$ ).

Now we can handle the hard case. Assume that $A$ is a vertex in $S_{i+1}$ but not a vertex in $S_{i}$, and $A$ is an inner point of $\left[S_{i+1}\right]=\left[S_{i}\right]$. We have to show that $S_{i}$ includes the the triangle $\tilde{F}$ (Fig. 11). By $(*)$ the star within $S_{i}$ centered at $B$ can be extended to a complete legal star $C$ by adding some tiles not overlapping $S_{i}$. As we have seen, $C$ includes the triangle $\tilde{F}$. If $\tilde{F} \operatorname{did}$ not belong to $S_{i}$, then $A$ would lie on the border of $\left[S_{i}\right]$, as $\tilde{F}$ does not overlap $S_{i}$.

(3) It suffices to prove the statement for stars $C_{1}$ only. Indeed, for every $i>1$ the star $C_{i}$ is obtained from $C_{1}$ by $i-1$ decompositions. Thus, if $C_{1}$ occurs in $S_{n}$, then $C_{i}$ occurs in $S_{n+i-1}$. There are four stars of the type $C_{1}$ : we have two ways to label the axis (yellow or blue) and two ways to choose its orientations. The stars $C_{1}$ with yellow axis of both orientations appear on the altitude of the supertile $S_{10}$ (Fig. 13 ). The stars $C_{1}$ with blue axis appear on the altitude of the supertile $S_{4}$ on Fig. 7 (they appear also in Fig. 13 , inside the supertile $S_{10}$ ).

Remark 4. We can explain now why we use 4 digital marks. Assume that digital marks belong to 


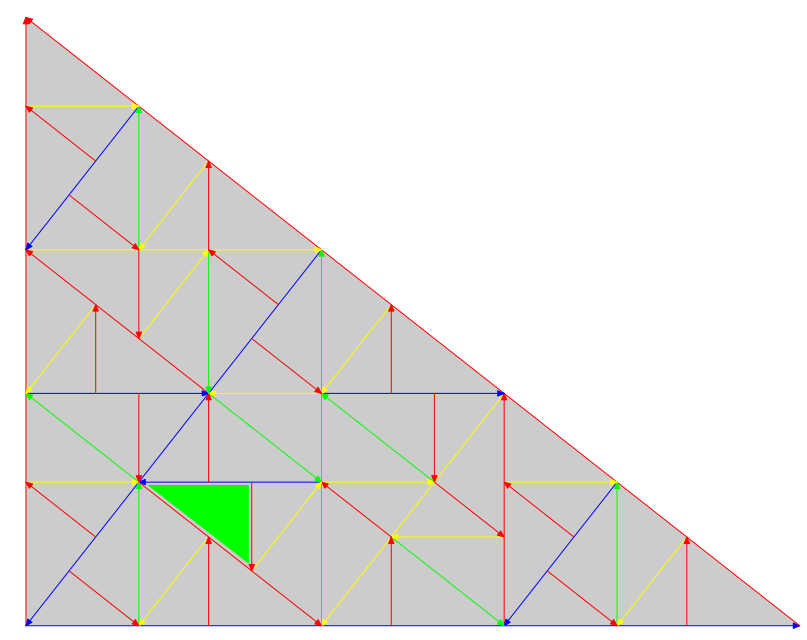

Figure 14: A colored supertile $S_{8}$ contains a large tile of the same color as the initial tile $S_{0}$ which it was obtained from.

$\mathbb{Z}_{k}=\{0,1, \ldots, k-1\}$, the substitution $\tilde{\sigma}$ works as before and the local rule $L_{k}$ stipulates that all stars occur in supertiles. Then we would have $\operatorname{lcm}(4, k)$ legal stars of the shape $C_{6}, C_{7}$. Indeed, geometrically, substitution acts as $90^{\circ}$ rotation on stars of this shape (see Fig. 10). And on digital marks it works as adding 1 . Thus we have two independent cycles of lengths 4 and $k$, whose superposition is a cycle of length $\operatorname{lcm}(4, k)$.

One can show that the choice $k=1,2$ does not work, as for $k=1,2$ there is a periodic tiling satisfying the local rule $L_{k}$. The choice $k=3$ might work. However, there are $\operatorname{lcm}(4,3)=12$ legal stars of the shape $C_{6}, C_{7}$ for that $k$, thus the local rule becomes too complicated any way. So the choice $k=4$ seems to be optimal.

Proof of Corollary 2. Consider the supertile of order 0 shown in Fig. 7. Decomposing that large tile 8 times, we obtain a supertile of order 8 which is an L-tiling by Theorem 1 (see Fig. 14). The green tile $A$ has the same color as the initial large tile. Thus, if we decompose this supertile $S_{8}$ eight times, then the green tile will produce another supertile $S_{8}$. In this way we can construct a tower of supertiles $S_{0} \subset S_{8} \subset S_{16} \subset \ldots$ whose union is an L-tiling of the entire plane.

Remark 5. One can show that there are only 7 different small tiles and 15 different large tiles (up to isometry) that occur in supertiles as inner tiles (a tile is called an inner tile of a supertile, if no its side lies on the border of the supertile). Thus we can reduce the total number of proto-tiles to 22. Indeed, to prove Corollary 2, we do not need remaining tiles.

A derivation of Theorem 4 from Theorem 3. By definition, every fragment of every substitution tiling of the plane occurs in a colored supertile, hence is legal by Theorem 1(2). Therefore every substitution tiling is an L-tiling.

To prove the converse, consider any fragment $P$ of an $L$-tiling $T$ of the plane. We have to show that $P$ occurs in a (colored) supertile. To this end add in $P$ a finite number of tiles from $T$ so that $P$ becomes an inner part of the resulting fragment $Q$. By Theorem 3 we can compose $T$ any number of times and the resulting tiling is an L-tiling. Consider the sets of the form $\sigma^{k} C$ where $C$ is a star within the tiling $\sigma^{-k} T$. As $k$ increases, these sets increase as well. If $k$ is large enough, 
then the set $Q$ is covered by a single such set, say by $\sigma^{k} C$, that is, $Q \subset \sigma^{k} C$ (Lemma 1 below). As $\sigma^{-k} T$ is an L-tiling, all its stars are legal. In particular, the star $C$ is legal. By Theorem 1(3), the star $C$ appears in a supertile, say in $S_{n}$. Therefore the tiling $\sigma^{k} C$ appears in the supertile $S_{n+k}$. Hence the patch $Q$ appears in that supertile provided we ignore labels and orientations of its outer sides. Since no side of the patch $P$ is an outer side of $Q$, we are done. It remains to prove Lemma 1 .

Lemma 1. If $k$ is large enough compared to $d$, then for any substitution tiling $T$ of the plane for any its $d$-fragment $P$ there is a star $C$ in the tiling $\sigma^{-k} T$ such that $\sigma^{k} C$ includes the entire patch $P$.

Proof. Consider supertiles of the form $\sigma^{k}\{A\}$ for $A \in \sigma^{-k} T$, call them $k$-supertiles. These supertiles partition $T$ and hence $P$ is covered by a finite number of $k$-supertiles. More specifically, $P$ is covered by those $k$-supertiles $\sigma^{k}\{A\}$ which intersect $[P]$. For small $k$, for instance for $k=0$, the respective tiles $A$ might not belong to a single star within the tiling $\sigma^{-k} T$. However, the sizes of $k$-supertiles increase as $k$ increases, and for a large enough $k$ the respective tiles $A$ belong to a single star within the tiling $\sigma^{-k} T$. Indeed, cover the set $[P]$ by a disc $S$ of radius $d$ (centered at any point from $[P])$. It suffices to show that if $k$ is large enough, then there is a star $C$ in $\sigma^{-k} T$ such that $\left[\sigma^{k} C\right]$ covers disc $S$. In other words, $[C]$ covers $H^{-k} S$, the inverse image of $S$ under the $k$ th power of the reference homothety $H$. The radius of $H^{-k} S$ equals $\phi^{k} d$, therefore the claim follows from the following

Geometrical observation: Let $\alpha$ denote the minimal angle of the right golden triangle and $h$ the length of the altitude of the small right golden triangle. Let $S$ be a disc of diameter $D$. If $h \geqslant D / \sin \alpha+D$, then every tiling of the plane has a star $C$ such that $S \subset[C]$.

Proof of the observation. We have to show that tiles intersecting the disc $S$ belong to a single star. If there is a single such tile, then this is obvious. If there are exactly two such tiles, $A$ and $B$, then they must share a part of a side and at least one end of these two sides belongs to both tiles. Then for the star $C$ centered at that end we have $[C] \supset A \cup B \supset S$. Finally, if there are three or more such tiles, then at least one of those tiles, call it $F$, has common points with $S$ lying on two different sides of the tile $F$. Let $E$ denote the common point of those sides and let $A, B$ denote the points from $S$ that belong to different sides of $F$. The angle $\angle A E B$ is one of the angles of the right golden triangle and the length of $A B$ is at most $D$. Hence $D \geqslant|A B| \geqslant|A E| \sin \alpha$. Therefore $|A E|$ is at most $D / \sin \alpha$. All the points from $S$ are at distance at most $D$ from $A$ and hence at distance at most $D / \sin \alpha+D$ from $E$. That is, $S$ is covered by the disc with center $E$ and radius $D / \sin \alpha+D$. That disc is covered by the star centered at $E$, provided the length of the altitude $h$ of small tiles is at least its radius $D / \sin \alpha+D$.

This observation provides the relation between $k$ and $d$ we need. Assume that $h \geqslant 2 d \phi^{k}(1 / \sin \alpha+$ 1 ). Then any $d$-fragment $P$ of the initial substitution tiling $T$ is covered by a disc of diameter $2 d$ and is included in $\sigma^{k} C$ for some star $C$ from the tiling $\sigma^{-k} T$.

Proof of Theorem 3. Let $T$ be an L-tiling of the plane. We have to show that it has a composition and that its composition is again an L-tiling.

Why $T$ has a composition? Let $S$ be any small tile from $T$. Consider the star within $T$ centered at the vertex of the right angle of $S$. We know that that star is legal. There are only two stars in 

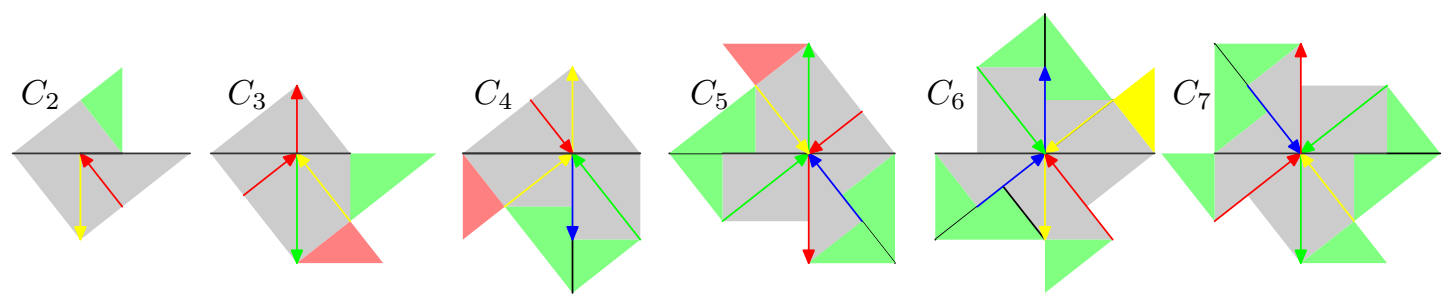

Figure 15: Surroundings of legal stars

the list of legal stars, whose center is a vertex of the right angle of a small tile, the stars $C_{1}$ and $C_{3}$.

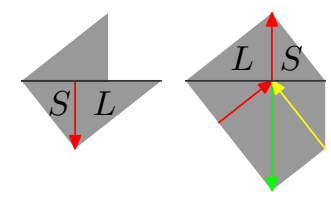

In both these stars the small tile $S$ has the complement labeled $L$ on the picture. Therefore the tiling $T$ has a composition which is obtained by removing the common legs of all complementary pairs of tiles $S, L$ and by decrementing all labels by 1 (and then applying $H^{-1}$, where $H$ is the reference homothety). Hypotenuses of large tiles are built of two legs of different tiles from $T$. Those legs have the same labels, since they belong to axes of stars $C_{1}$ and $C_{3}$. Hence hypotenuses of all large tiles obtain unique digital labels. Finally, new labeling of sides is legal, that is, the hypotenuses and small legs of large tiles and large legs of small tiles have even colors and all other sides have odd colors.

Why the composition of $T$ is an L-tiling? We have to show now that the resulting colored tiling is an $L$-tiling. Since $T$ satisfies item (1) of the local rule, so does $\sigma^{-1} T$. Let us verify item (2) of the local rule.

Let $A$ be a vertex of a tile from $\sigma^{-1} T$. We have to prove that the colored star centered at $A$ in $\sigma^{-1} T$ is legal. First note that the star centered at the same vertex $A$ in the initial tiling $T$ is different from $C_{1}$, as the centers of stars $C_{1}$ become inner points of sides in $\sigma^{-1} T$. Thus that star is one of the stars $C_{2}-C_{7}$. We claim that the composition transforms these stars by the inverse arrows from Fig. 10. To prove this, we need the following

Lemma 2. If $T$ is an L-tiling of the plane, then every its star of type $C_{2}-C_{7}$, depending on its index, includes all tiles marked green in Fig. 15 and does not include tiles marked red (the star itself is marked grey).

We will first finish the proof of the theorem assuming Lemma 2, Lemma 2 guarantees that via composition tiles from each star $C_{i}$ except $C_{6}$ are transformed to tiles from the star $C_{i-1}$. In the course of composition, we decrement the labels and do not change the orientation of sides. Therefore, digital labels and orientations of all sides become, as in the star $C_{i-1}$. Hence for $i>1$, $i \neq 6$, the star $C_{i}$ is transformed to the star $C_{i-1}$. For the star $C_{6}$, one its large tile can be transformed in two ways, depending on whether the small yellow tile is in $T$ or not. If it is, then tiles from $C_{6}$ form the star $C_{7}$ and otherwise $C_{5}$.

Hence in the course of composition, all stars in the tiling $T$ are transformed by the inverse grey arrows from Fig. 10, which implies legality of all stars in $\sigma^{-1} T$. It remains to prove Lemma 2 . 


\section{Proof of Lemma 2}

We first show that in any L-tiling of the plane every star must have some fixed neighborhood, called the neighborhood of the star. Those neighborhoods include all green small tiles (Fig. 15) except one small tile near $C_{7}$. In this analysis, we do not use labels and orientation of sides of tiles. It is instructive, for reader's convenience, to print out all the legal stars and their neighborhoods (Fig. 38, 39 and 40 on pages 29 and 30) and then to cut them out of paper. Matching tiles from those paper stars with the tiles from the figures below, it is easy to verify all the claims that certain stars do not fit in certain places.

The neighborhoods of legal stars. The neighborhoods of the stars $C_{1}, C_{2}, C_{3}, C_{4}, C_{5}$ are shown in Fig. 16. They all are centrally symmetric. The initial star is colored in grey, the added tiles are colored in light-grey. These neighborhoods are obtained from each other by decomposition. One can verify that each of the first five stars indeed must have such neighborhood as follows.

The star $C_{1}$. Look at the blue vertex inside the grey star $C_{1}$ (Fig. 16). That vertex lies on the large leg of a large tile. One can easily verify that there is the unique legal star whose center lies on the large leg of a large tile, namely the star $C_{1}$. Hence the star within $T$ centered at the blue vertex is again $C_{1}$ and we get the sought neighborhood.
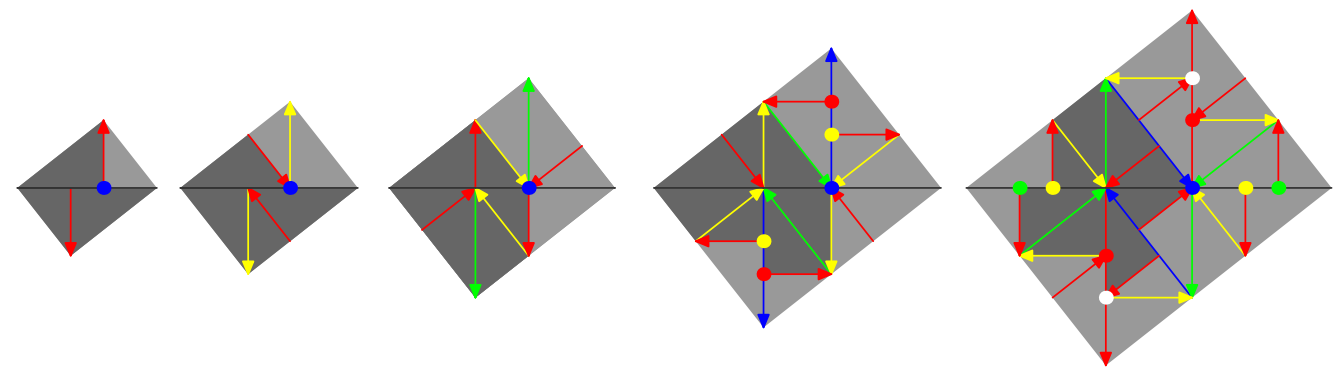

Figure 16: The neighborhoods of the first five stars.

The star $C_{2}$. The argument is similar to the previous one. The star $C_{2}$ has the following feature: it has a vertex (colored in blue) that lies on the hypotenuse of its large tile. It is easy to verify that there is the unique star whose center lies on the hypotenuse of its large tile, namely the star $C_{2}$.

The star $C_{3}$. The star $C_{3}$ is the unique star that has two small triangles sharing the small leg. Hence the star centered at the blue vertex is again $C_{3}$.

The star $C_{4}$. The star $C_{4}$ is the unique star that has two large triangles sharing the small leg. Hence the star centered at the blue vertex is again $C_{4}$. However this star does not complete the neighborhood: the stars centered at yellow vertices must be $C_{1}$ and the stars centered at red vertices again must be $C_{1}$.

The star $C_{5}$. The star $C_{5}$ is the unique star that has two small triangles sharing the hypotenuse. Hence the star centered at the blue vertex again must be $C_{5}$. The stars centered at yellow and green vertices must be $C_{1}$. Furthermore, the stars centered at red and white vertices must be $C_{2}$.

The neighborhoods of the stars $C_{6}, C_{7}$ are shown on Fig. 17 (on the right). One can verify in the following way that the stars $C_{6}, C_{7}$ indeed must have such neighborhoods.

The star $C_{6}$. The stars centered at yellow and green vertices must be $C_{1}$ and the stars centered at red and black vertices must be $C_{2}$ (on the left in Fig. 17). Now we see that the star centered at 

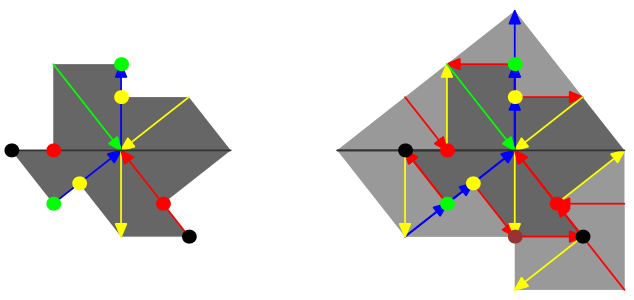

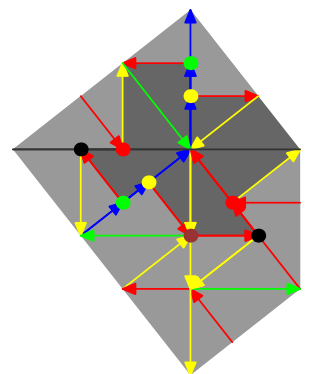

$C_{6}$

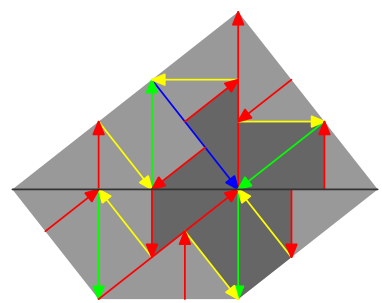

$C_{7}$

Figure 17: The neighborhood of the stars $C_{6}, C_{7}$.
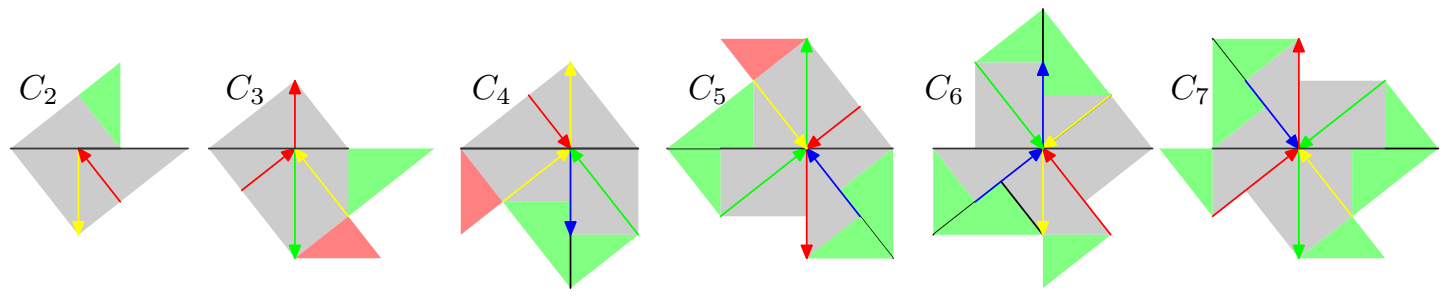

Figure 18: Neighborhoods of stars

the brown vertex must be $C_{3}$, which is added together with its neighborhood.

The star $C_{7}$. Since the star $C_{7}$ can be obtained from $C_{6}$ by rotation (ignoring labels and orientation), the arguments are entirely similar to those for the star $C_{6}$.

Now we can start the proof of the lemma. Assume that the star centered at a vertex $A$ in an L-tilling $T$ is $C_{i}$ where $i>1$. We have to prove that $T$ includes all tiles marked green on Fig. 18 (= Fig. 15) and does not include tiles marked red (the star itself is marked grey). We will treat all $i$ 's separately. We start with simple cases $i=2,5,6$.

The star of $A$ in $T$ is $C_{2}$ or $C_{6}$. It is easy to verify that in both cases the neighborhood of the star includes all tiles marked green in Fig. 18 , and we are done.

The star of the vertex $A$ in $T$ is $C_{5}$. Fig. $19(a, b)$ show the star $C_{5}$ and its neighborhood. We can see that the neighborhood includes all 4 tiles marked green in Fig. 18 . We need to show that the small red triangle is not in $T$. For the sake of contradiction assume that the tiling $T$ includes the patch shown in Fig. 19(c). We will say that a star $C_{i}$ fits for a given patch in a given its vertex if one can draw (an isometric copy of) $C_{i}$ centered at that vertex so that each of its tiles either does not overlap all the tiles from that tiling, or belongs to that tiling. It is easy to verify that only the star $C_{3}$ fits for this patch in the yellow vertex. Adding to the patch that star and its neighborhood, we obtain the patch shown in Fig. 19(d). Now, we can verify that no legal star fits for this patch in the blue vertex. Indeed, that star must have 7 triangles, and only $C_{4}$ has this property among legal stars. It is easy now to verify that $C_{4}$ does not fit. 


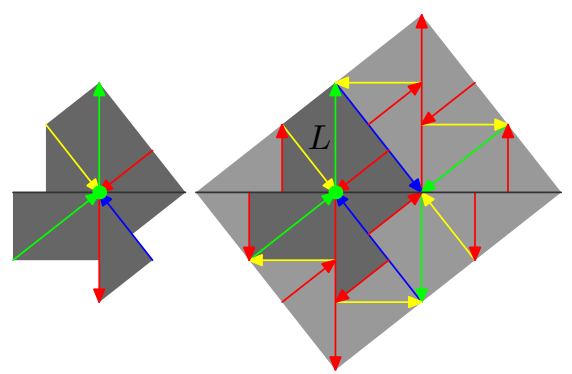

(a)

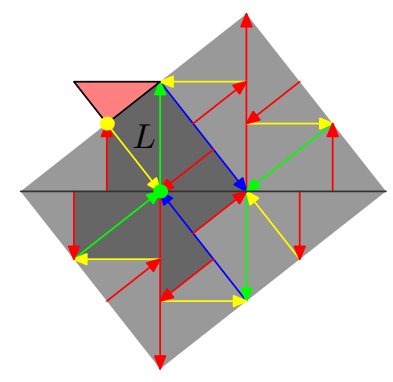

(c)

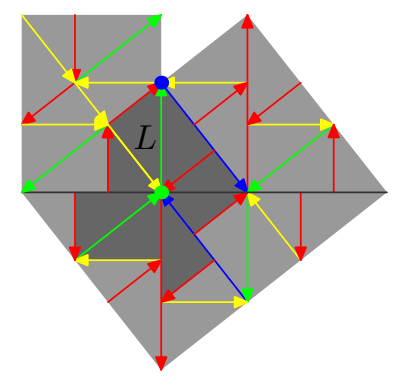

(d)

Figure 19: Composition of the star $C_{5}$

We proceed now to hard cases $C_{3}, C_{5}, C_{7}$. The arguments are very similar to those used in the case of $C_{5}$ but the analysis is much more involved. Therefore we moved most of the proof to Appendix, as the proof of Claim 1 (page 20.

The star of $A$ in $T$ is $C_{3}$. The vertex $A$ is colored green on Fig. 20(a) and its star is colored in dark-grey. In Fig. 20(b) we show the neighborhood of that star (added tiles are colored in lightgrey). We can see that the neighborhood includes the large tile marked green in Fig. 18. Now we have to show that the tiling $T$ does not the small triangle marked red in Fig. 20(c). This is the statement of Claim 1(a).

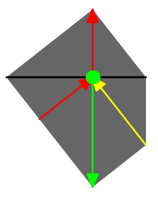

(a)

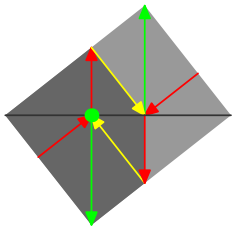

(b)

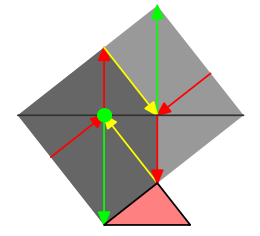

(c)

Figure 20: Composition of the star $C_{3}$.

The star of the vertex $A$ is $C_{4}$. The vertex $A$ is shown by the green point in Fig. 21(a). And Fig. 27(b) shows the neighborhood of that star. We can see that the neighborhood includes both tiles marked green in Fig. 18. It remains to show that the tiling $T$ does not the small triangle marked red (Claim 1(b)).

The star of $A$ in $T$ is $C_{7}$. Fig. 22(a,b) show the star $C_{7}$ and its neighborhood. We can see that the neighborhood includes all 6 tiles marked green in Fig. 18, except for the bottommost small tile. To prove that it includes also that tile, consider the blue vertex. Only the stars $C_{2}, C_{3}, C_{4}$ fit for the patch in that vertex, they are shown on Fig. 22(c). If the star of the blue vertex is $C_{3}$, we are 


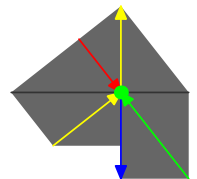

(a)

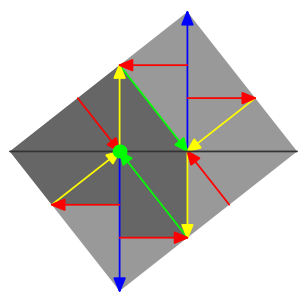

(b)

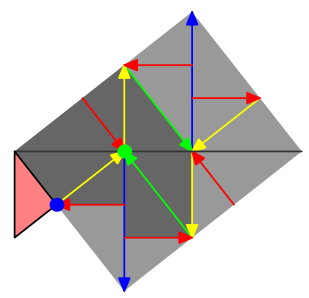

(c)

Figure 21: Composition of the star $C_{4}$.

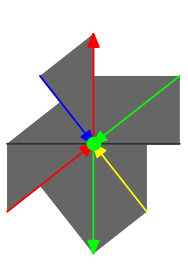

(a)

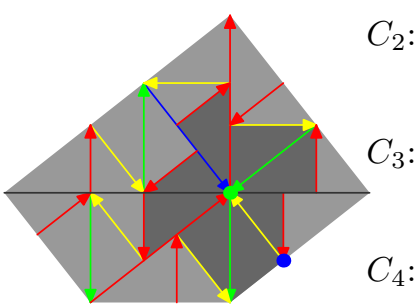

(b)

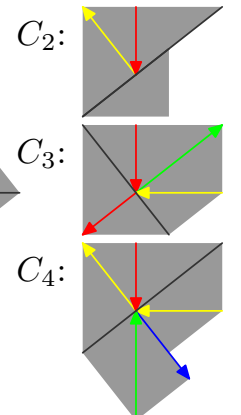

(c)

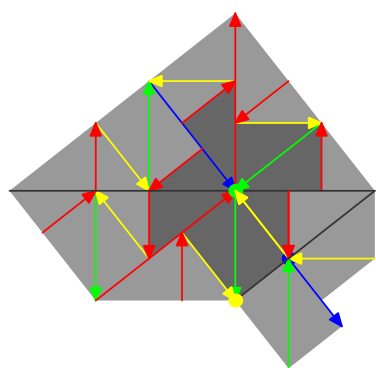

(d)

Figure 22: Composition of the star $C_{7}$.

done, as that star includes the sought tile. It remains to show that neither of the stars $C_{2}, C_{4}$ can stand in the blue vertex.

It is easy to show that $C_{4}$ cannot be there. Indeed, adding that star to the patch, we obtain the patch shown in Fig. 22(d). No legal star fits for that patch in the yellow vertex. And Claim 1(c) claims that the star centered at the blue vertex cannot be $C_{2}$ either. It remains to prove

Claim 1. The following patches (Fig. 23) cannot occur in L-tilings of the plane.

To prove the claim, we explore a small neighborhood of the patches in a similar way, as it was done in finding neighborhoods of the stars. The proof is deferred to Appendix.

\section{Acknowledgments}

The author is grateful to Daria Pchelina and Alexander Kozachinskii for verifying the proofs and reading the preliminary version of the paper, and to anonymous referees for valuable comments.

\section{References}

[1] M. Baake, U. Grimm, Aperiodic Order. Vol 1: A Mathematical Invitation. Cambridge University Press, 2013. 


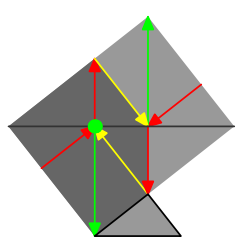

(a)

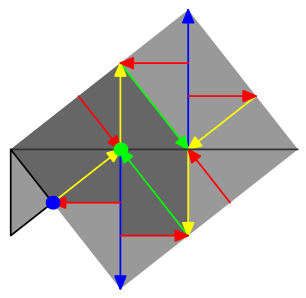

(b)

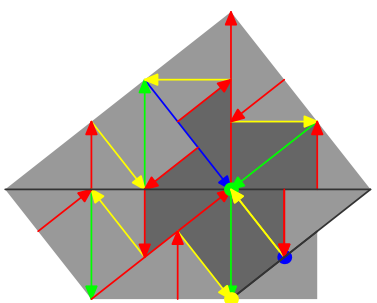

(c)

Figure 23: The following patches do not occur in L-tilings of the plane (Claim 1)

[2] L. Danzer and G. van Ophuysen. A species of planar triangular tilings with inflation factor $\sqrt{-\tau}$. Res. Bull. Panjab Univ. Sci. 2000, 50, 1-4, pp. 137-175 (2001)

[3] B. Durand, L. Levin, A. Shen. Local rules and global order, or aperiodic tilings. The Mathematical Intelligencer, Volume 27, Issue 1, pp 64-68 (2005).

[4] B. Durand, A. Shen , N. Vereshchagin. On the Structure of Ammann A2 Tilings. Discrete and Computational Geometry, vol. 63, pp. 577-606 (2020).

[5] T. Fernique and N. Ollinger. Combinatorial substitutions and sofic tilings. Journees Automates Cellulaires 2010, J. Kari ed., TUCS Lecture Notes 13 (2010), 100-110.

[6] C. Goodman-Strauss, Matching Rules and Substitution Tilings. Annals of Mathematics 147 (1998) 181-223.

[7] B. Grünbaum, G. C. Shephard, Tilings and Patterns. Freeman, New York 1986.

[8] D. Frettlöh, E. Harriss, F. Gähler, Tilings encyclopedia, https://tilings.math.uni-bielefeld.de/

[9] D. König. Theorie der Endlichen und Unendlichen Graphen: Kombinatorische Topologie der Streckenkomplexe (in German). Leipzig: Akad. Verlag, 1936.

[10] S. Mozes, Tilings, substitution systems and dynamical systems generated by them. J. Analyse Math. 53 (1989), 139-186.

[11] R. Penrose, Pentaplexity. Eureka, 39: 16-22 (1978)

[12] R. Robinson, Undecidability and nonperiodicity of tilings in the plane. Invent. Math., 12 (1971), 177-209

[13] B. Solomyak, Nonperiodicity implies unique composition for self-similar translationally finite tilings. Discrete and Computational Geometry 20 (1998) 265-279.

[14] N. Vereshchagin. Aperiodic Tilings by Right Triangles. In: Descriptional Complexity of Formal Systems - 16th International Workshop, DCFS 2014, Turku, Finland, August 5-8, 2014. Proceedings. Lecture notes in computer sciences, Vol. 8614. Berlin : Springer Verlag, 2014. P. $29-41$. 


\section{A Proof of Claim 1}

(a) For the sake of the contradiction assume that the patch in Fig. 24(a) occurs in an L-tiling. Look at the vertex colored black. A quick look at the list of legal stars reveals that only the star

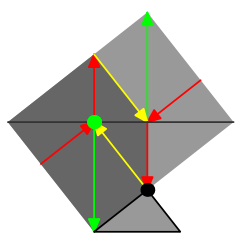

(a)

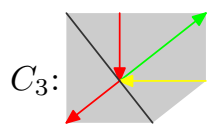

(b)

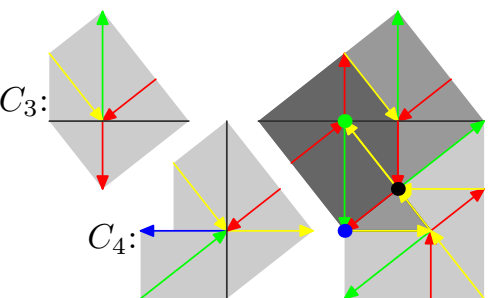

(c)

(d)

Figure 24: Composition of the star $C_{3}$ (the beginning).

$C_{3}$ (shown in Fig. 24(b)) fits for the patch in that vertex. Adding that star and its neighborhood, we obtain the patch shown in Fig. 24(d), where the added tiles are colored light-gray.

Now look at the blue vertex on the bottom left. In that vertex only the stars $C_{3}$ and $C_{4}$ fit. Those stars are shown in Fig. 24(c). The star $C_{3}$ has non-matching orientation of the green arrow, hence $C_{3}$ cannot be the star within $T$ in the blue vertex. Therefore, it is the star $C_{4}$. Adding the star $C_{4}$ and its neighborhood, we obtain the tiling shown on Fig. 25(a).

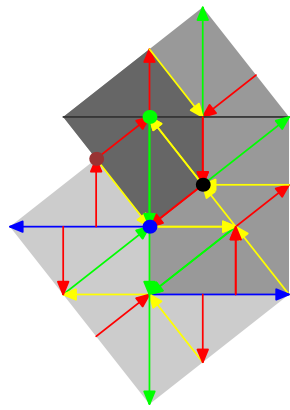

(a)

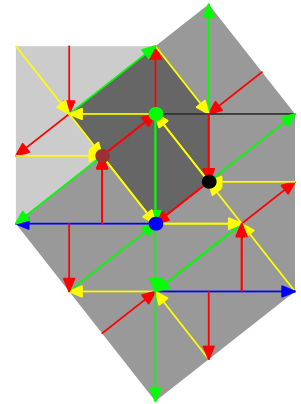

(b)

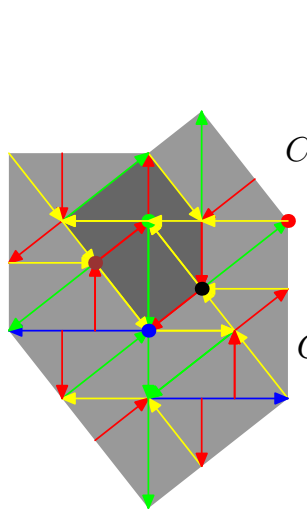

(c)

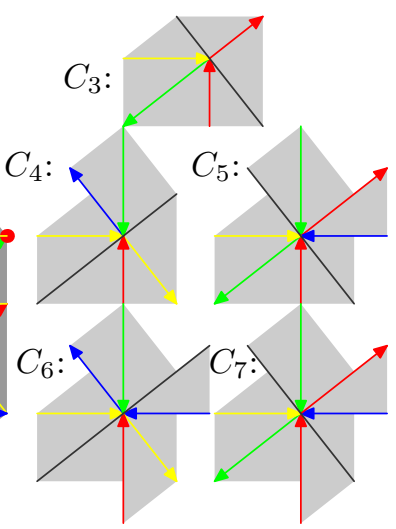

(d)

Figure 25: Composition of the star $C_{3}$ (the end).

A small search reveals that only the star $C_{3}$ fits for that patch in the brown vertex on the top left. Fig. 25(b) shows the tiling that is obtained by adding the star $C_{3}$ and its neighborhood. Thus we conclude that the axis of the initial star and its extension to the right must be directed from the right to the left (yellow arrows in Fig. 25(c)). Now look at the beginning of the leftmost yellow arrow (the red point on the top right in Fig. 25(c)). Only the stars shown in Fig. 25(d) fit for the resulting patch in the red vertex. However none of them can be there, since all they have non-matching orientation of the horizontal yellow arrow.

(b) For the sake of contradiction assume that an L-tiling $T$ includes the patch shown in Fig. 
26(a). Our plan is the following. We first show that, in addition to all tiles in the patch in Fig. 26(a),

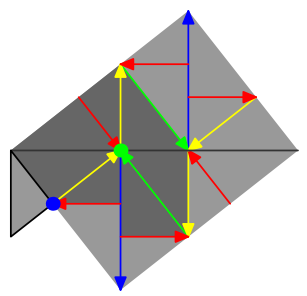

(a)

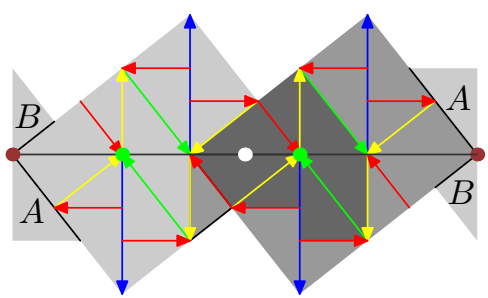

(b)
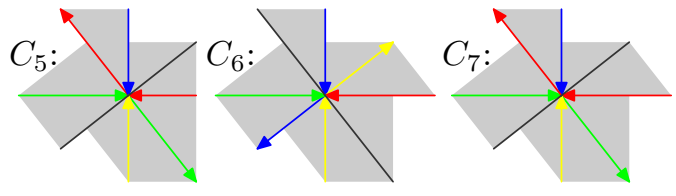

(c)

Figure 26: Composition of the star $C_{4}$ (beginning)

the tiling $T$ must contain all the tiles shown in Fig. 26(b).

Assume that this is done. It is easy to verify that only the stars $C_{5}, C_{6}, C_{7}$ (shown in Fig. 26(c)) fit for the patch in the rightmost brown vertex. In all three cases the axis of the initial star (the horizontal black line) must be directed rightwards. However, similar arguments applied to the leftmost brown vertex show that that axis must be directed leftwards, which is a contradiction.

So we have to show that the tiling $T$ must contain all the tiles shown in Fig. 26(b). To this end, let us go back to Fig. 26(a). We copied that patch in Fig. 27(a). Only the star $C_{3}$ fits for this

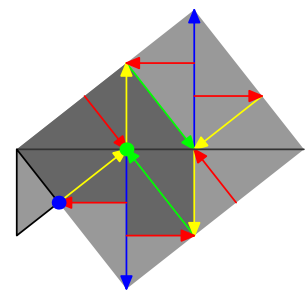

(a)

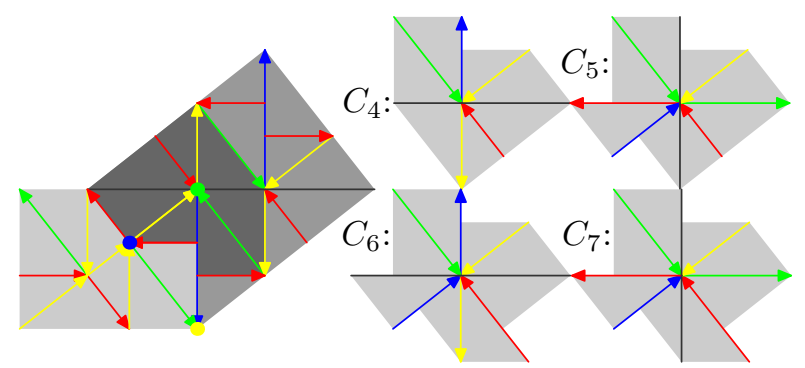

(b)

(c)

Figure 27: Composition of the star $C_{4}$ (continued)

patch in the blue vertex. Fig. 27(b) shows the patch that is obtained by adding the star $C_{3}$ and its neighborhood. Look now at the yellow vertex on the bottom. Only the stars $C_{4}, C_{5}, C_{6}, C_{7}$ fit for the patch in that vertex, they are shown in Fig. 27(c). Note that the stars $C_{4}, C_{6}$ (from the left column) have non-matching orientation of the vertical blue arrow, which must direct downwards, hence cannot be there. We will consider the remaining two cases separately.

Case 1: the star $C_{7}$ is in the yellow vertex in Fig. 27(b). In this case we are able to derive a contradiction quite easily. Adding the star $C_{7}$ and its neighborhood we obtain the patch shown in Fig. 28(a). Only the stars $C_{3}, C_{4}$ (shown in Fig. 28(b)) fit in the blue vertex. Adding the star $C_{4}$, we obtain the patch shown in Fig. 28(c). We can see that no legal star fits in the green vertex on the right.

Hence only the star $C_{3}$ can be in the blue vertex. Adding that star and its neighborhood, we obtain the patch shown in Fig. 29(b). Only the stars $C_{3}, C_{4}$ (shown in Fig. 29(c)) fit for the resulting patch in the green vertex on the right. However the star $C_{3}$ has non-matching orientation 


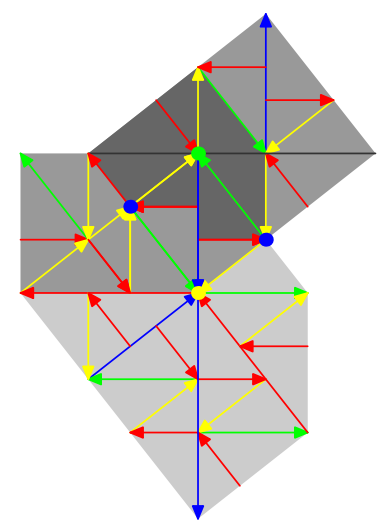

(a)

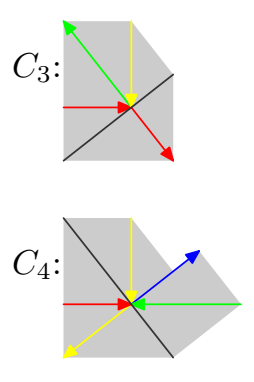

(b)

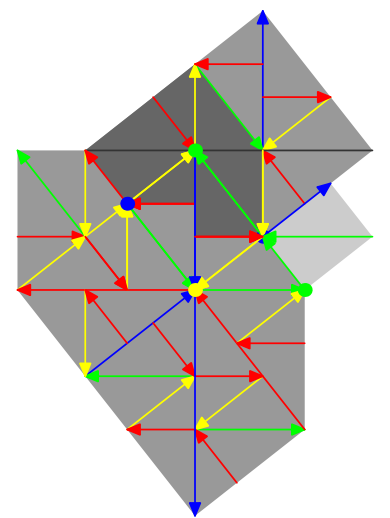

(c)

Figure 28: Composition of the star $C_{4}$ : case 1 (beginning)

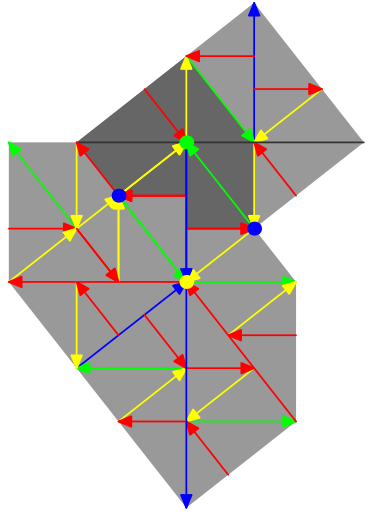

(a)

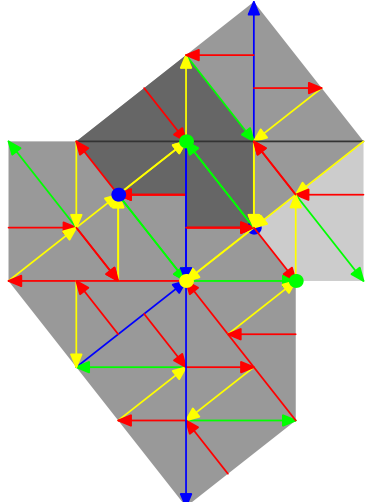

(b)
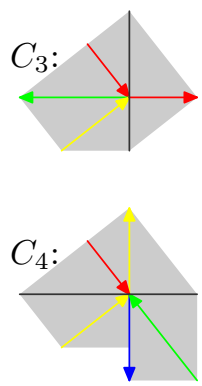

(c)

Figure 29: Composition of the star $C_{4}$ : case 1 (continued)

of the green arrow, hence the star centered at the green vertex is $C_{4}$. Adding it and its neighborhood, we obtain the patch in Fig. 30(a).

Only the stars $C_{4}, C_{5}, C_{6}, C_{7}$ (shown in Fig. 30(b)) fit for the patch in the brown vertex on the top right. However all those stars have non-matching orientation of the yellow arrow in the lower half of the star. Thus we have derived a contradiction in the first case.

Case 2: the star centered at yellow vertex in Fig. 27(b) is $C_{5}$. In this case we need a more involved analysis. Let us go back to Fig. 27(b) and add the star $C_{5}$ and its neighborhood in the yellow vertex. We obtain the patch shown on Fig. 31(b). Which stars fit for the patch in the leftmost vertex (colored in red)? These are the stars $C_{6}, C_{7}$, which are shown in Fig. 31(a). Assume first that it is $C_{6}$. Adding the neighborhood of the star $C_{6}$ centered at the red point, we get the patch shown in Fig. 31(c). If it is $C_{7}$, we get a patch that differs from this one in orientation and labels of some sides. This difference does not matter and therefore we will consider only the case of $C_{6}$.

Look at the vertex colored black (on the top left). Only the star $C_{4}$ fits for the patch in 


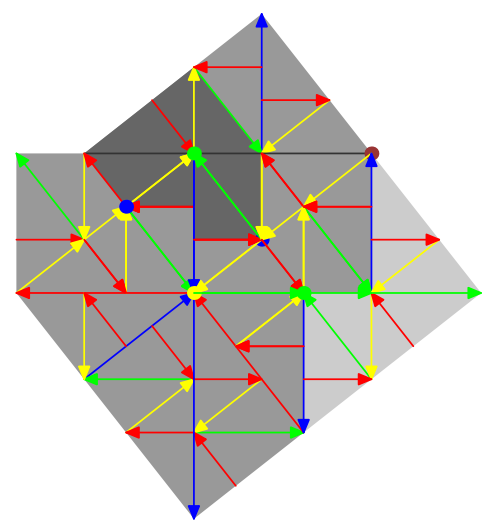

(a)
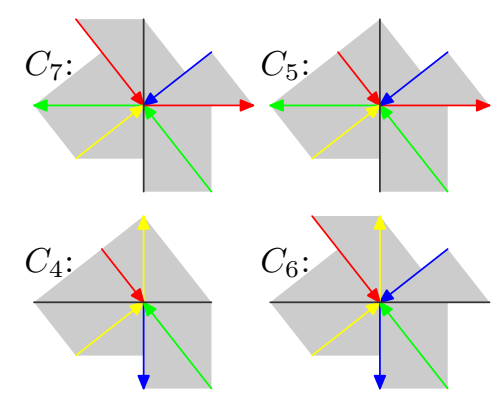

(b)

Figure 30: Composition of the star $C_{4}$ : case 1 (the end)

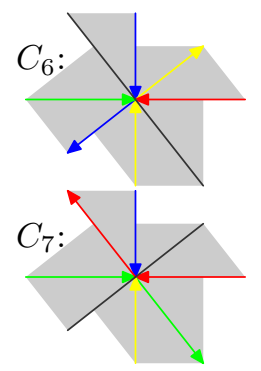

(a)

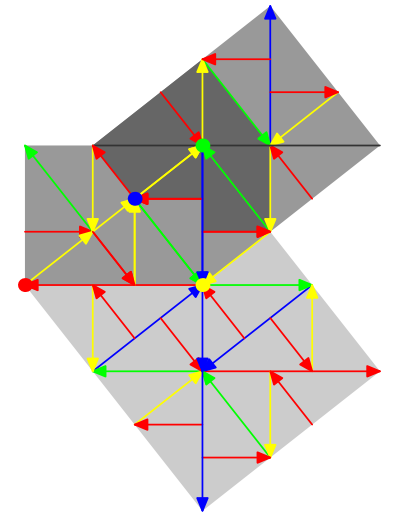

(b)

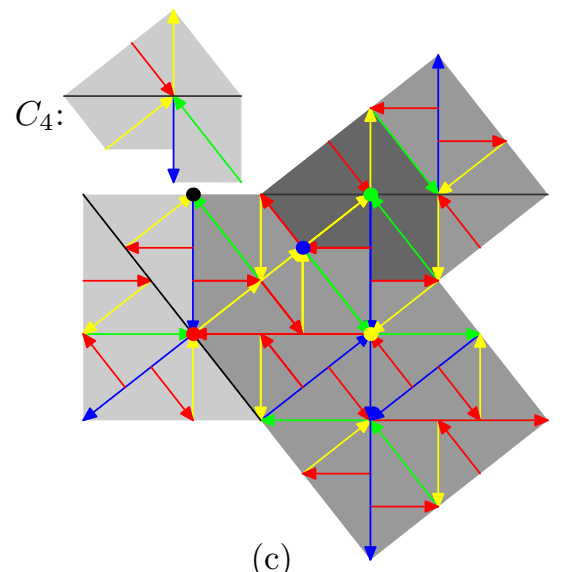

(c)

Figure 31: Composition of the star $C_{4}$ : case 2 (beginning)

that vertex. Adding $C_{4}$ and its neighborhood in the black vertex we obtain the patch shown in Fig. 32(a). We have shown that our tiling $T$ includes all the tiles from Fig. 26(b) except for both triangles labeled by letter $B$ and one triangle labeled by letter $A$. We have also shown that the tiling $T$ includes the image of the initial star under the inversion through the white point. Via central symmetrical arguments we can prove that $T$ includes also the other triangle labeled by letter $A$ in Fig. 26(b). It remains to show that $T$ includes both triangles labeled by $B$.

To this end look at the blue vertex on the right in Fig. 32 (a). Only the stars $C_{3}$ and $C_{4}$, shown in Fig. 32(b), fit for the patch in that vertex. If the star centered at the blue vertex is $C_{3}$, we obtain the patch shown in Fig. 32(c), and no legal star fits for it in the green vertex on the right.

In the remaining case the star centered at the blue vertex is $C_{4}$, and we get the patch shown in Fig. 33(a). Adding its neighborhood, we get the patch that includes the sought triangle $B$ (see Fig. 33(b)). Via central symmetrical arguments we can prove that $T$ includes also the other triangle labeled by letter $B$. We have reached our goal: we have proved that the tiling $T$ includes 


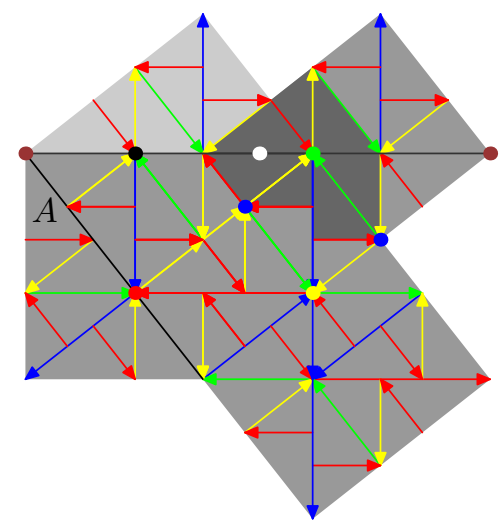

(a)

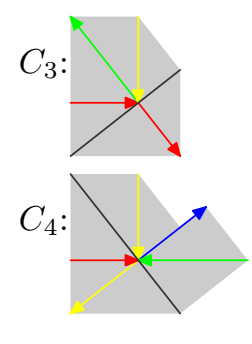

(b)

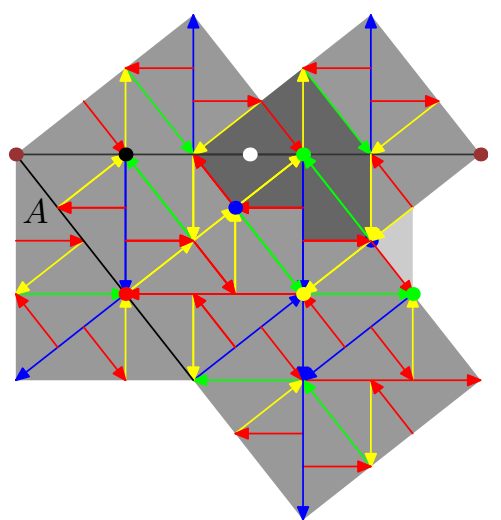

(c)

Figure 32: Composition of the star $C_{4}$ : case 2 (continued)

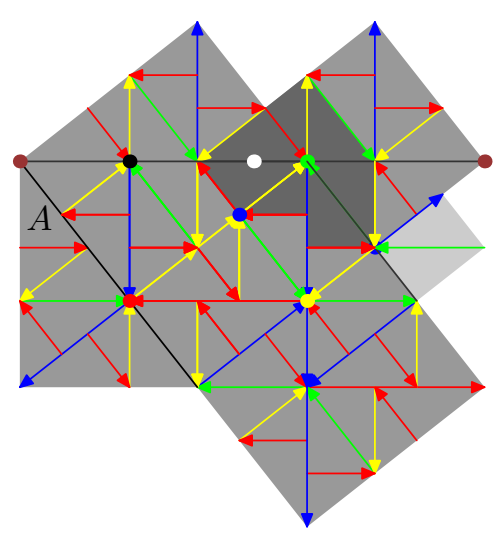

(a)

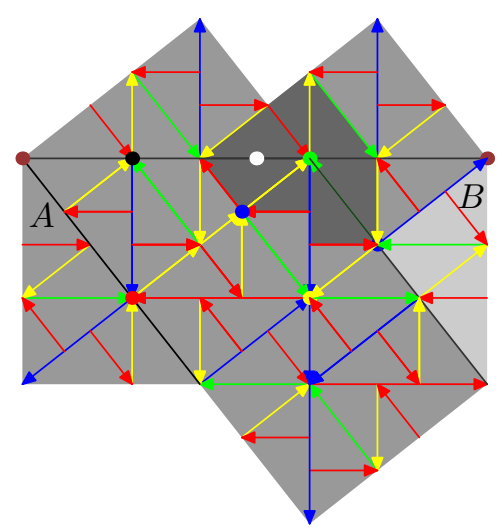

(b)

Figure 33: Composition of the star $C_{4}$ : case 2 (the end) the patch shown in Fig. 26(b). 
(c) For the sake of contradiction assume that an L-tiling includes the patch shown in Fig. 34(a). First add the neighborhood of the star $C_{2}$ centered at the blue vertex, we obtain Fig. 34(b). Only

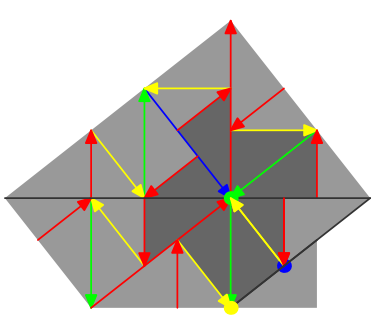

(a)

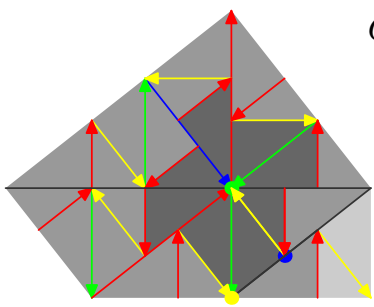

(b)

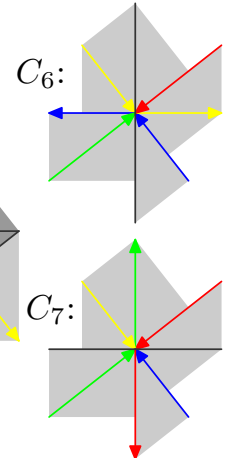

(c)

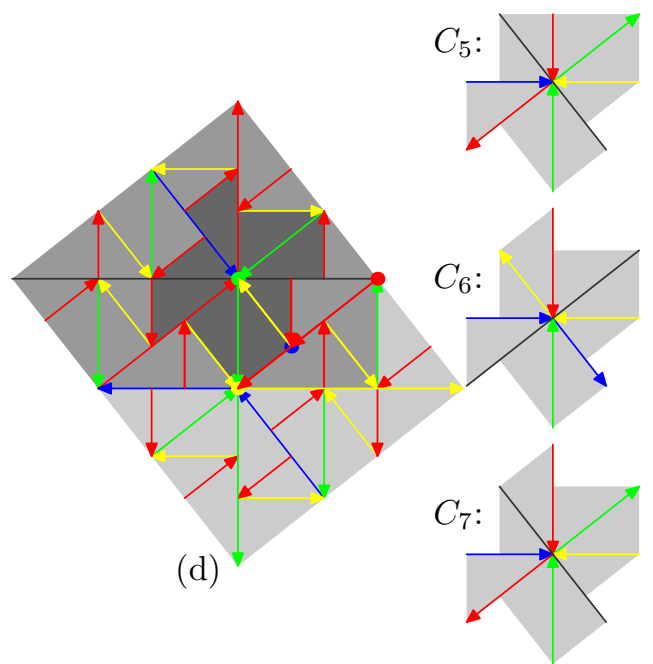

(e)

Figure 34: Composition of the star $C_{7}$ (continued)

the stars $C_{6}$ and $C_{7}$, shown on Fig. 34(c), fit for the resulting patch in the yellow vertex (on the bottom). However the star $C_{7}$ cannot be there, since its vertical green arrow has the non-matching orientation. Hence the star of the yellow vertex is $C_{6}$. Fig. 34(d) shows the patch which is obtained by adding that star and its neighborhood. Now look at the red vertex on the right. Only the stars $C_{5}, C_{6}, C_{7}$ (Fig. 34(e)) fit for the patch in that vertex. In all the three cases there is a horizontal blue arrow that goes into the red vertex. That arrow lies on the axis of the initial star.

Now we know the color and orientation of that axis (see Fig. 35(a)). We can find now the

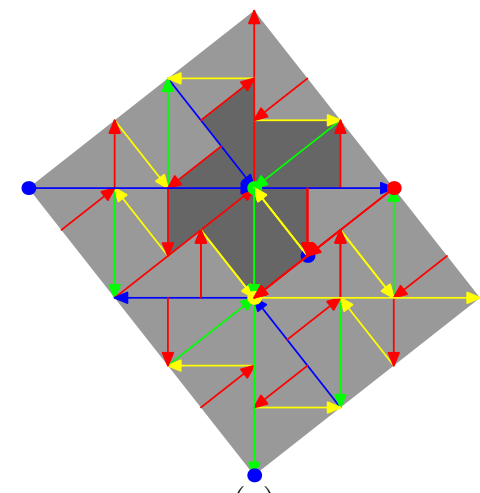

(a)
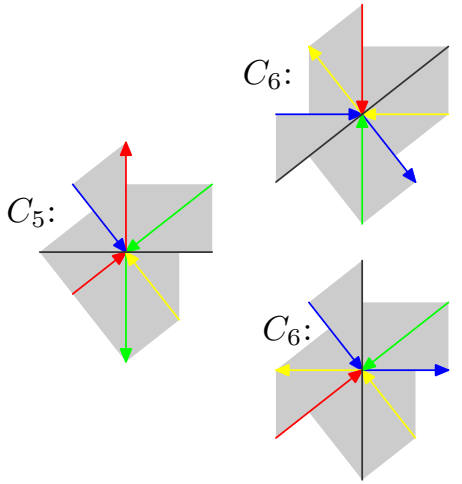

(b)

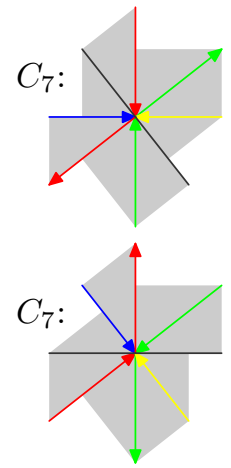

Figure 35: Composition of the star $C_{7}$ (continued)

stars in the leftmost and the bottommost vertices (both are colored blue). Indeed, only the stars 
$C_{5}, C_{6}, C_{7}$ fit for the patch in the bottommost vertex, and two latter stars fit in two ways (see Fig. 35(b)). In four cases the vertical arrow has the red (and not green) color. Hence those cases are impossible and only the lower star $C_{6}$ can stand in the bottommost blue vertex.

A similar situation occurs in the leftmost blue vertex: the stars $C_{3}, C_{4}, C_{5}, C_{6}, C_{7}$ fit for the patch there (two of them fit in two ways, see Fig. 36(a)). However only one star, the lower $C_{7}$, can

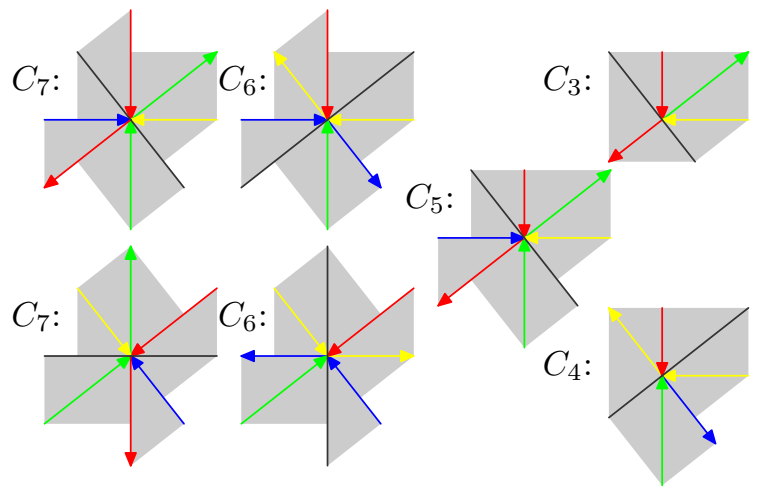

(a)

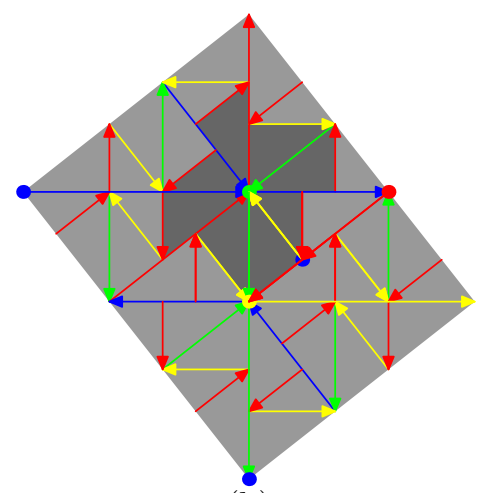

(b)

Figure 36: Composition of the star $C_{7}$ (continued)

have the matching color (blue) of the horizontal arrow.

Adding to the patch the stars in the blue vertices and adding then their neighborhoods, we obtain the patch shown in Fig. 37(b). Only the star $C_{4}$ fits for that patch in the yellow vertex,

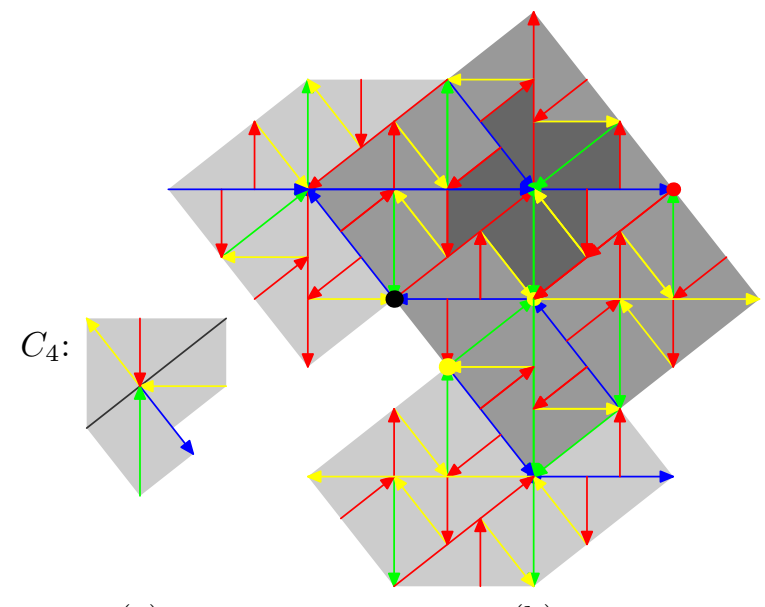

(a)

(b)

Figure 37: Composition of the star $C_{7}$ (the end)

that star is shown in Fig. 37(a). Now it is obvious that neither of the legal stars can stand in the adjacent black vertex, since the yellow arrow that goes out the yellow vertex into the black one cannot change its color to blue in the black vertex. We have considered all the cases. The claim is 
proved.

\section{B To cut out of paper}
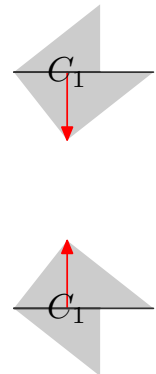
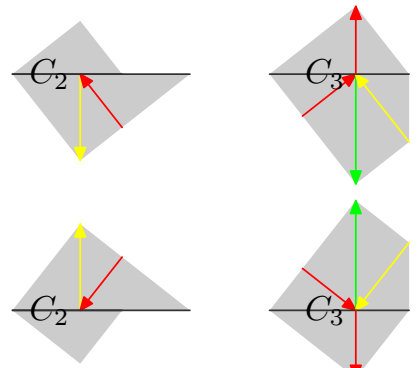
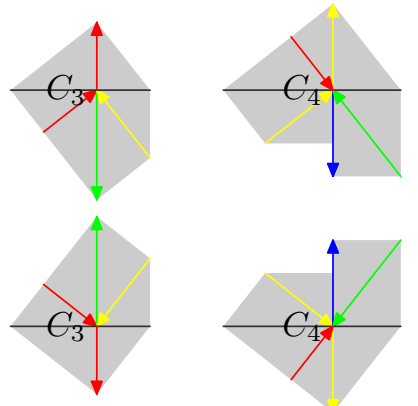
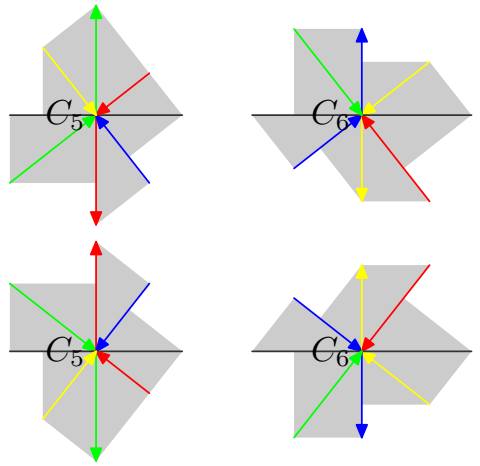

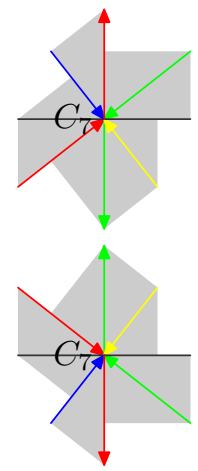

Figure 38: Legal stars. The stars in the second row are reflections of the stars in the first row. It is not necessary to use the color print, since labels on sides are used only in one place of the proof (in the very end).
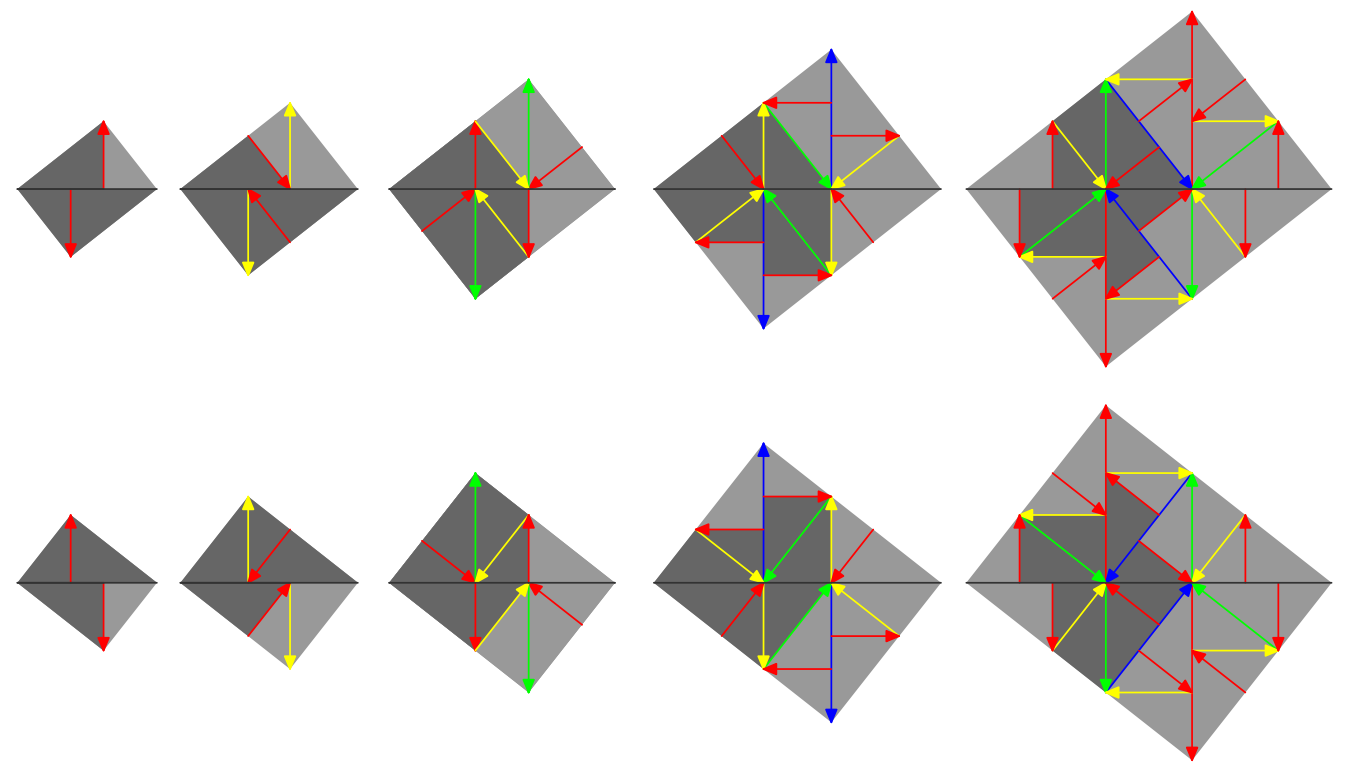

Figure 39: The neighborhood of the first five stars. 

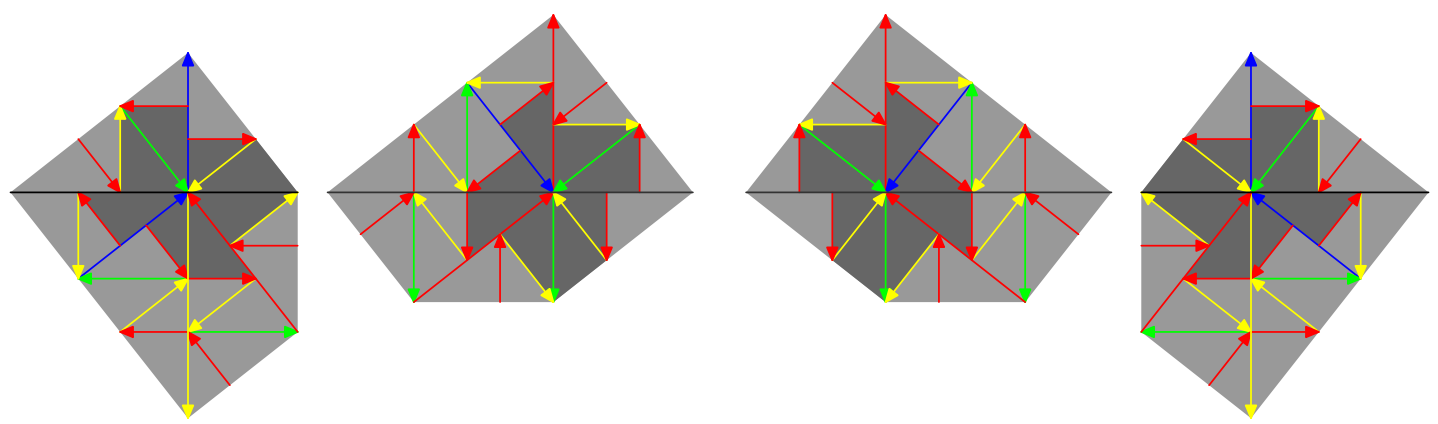

Figure 40: The neighborhoods of the stars $C_{6}, C_{7}$. 